\title{
MODELS OF PA: WHEN TWO ELEMENTS ARE NECESSARILY ORDER AUTOMORPHIC
}

\author{
SAHARON SHELAH
}

\begin{abstract}
We are interested in the question of how much the order of a nonstandard model of PA can determine the model. In particular, for a model $M$, we want to characterize the complete types $p(x, y)$ of non-standard elements $(a, b)$ such that the linear orders $\{x: x<a\}$ and $\{x: x<b\}$ are necessarily isomorphic. It is proved that this set includes the complete types $p(x, y)$ such that if the pair $(a, b)$ realizes it (in $M)$ then there is an element $c$ such that for all standard $n, c^{n}<a, c^{n}<b, a<b c$ and $b<a c$. We prove that this is optimal, because if $\diamond_{\aleph_{1}}$ holds, then there is $M$ of cardinality $\aleph_{1}$ for which we get equality. We also deal with how much the order in a model of PA may determine the addition.
\end{abstract}

\section{$\S 0$. INTRODUCTION}

Let $M$ be a model of Peano Arithmetic (PA). For an $a \in M$, by $M_{<a}$ we denote the set $\{c \in M: M \models c<a\}$ with the inherited linear order. For any pair $(a, b)$ of non-standard elements of $M$, let $(*)_{M, a, b}$ be the condition dfeined by

$(*)_{M, a, b}\left(M_{<a},<_{M}\right) \cong\left(M_{<b},<_{M}\right)$.

We will also consider

$(*)_{M, a, b}^{\mathrm{pot}}$ for every $N$, if $M \prec N$, then $(*)_{N, a, b}$

$(*)_{M, a, b}^{\mathrm{tp}}$ for every model $N$, if $M \equiv N$ and for some $M_{0} \prec N$ we have $\{a, b\} \subseteq M_{0} \prec$ $M$, then $(*)_{N, a, b}$.

Looking recently at models of PA, we wonder:

Question 0.1. What is the set of complete types $p(x, y)$ such that: if the pair $(a, b)$ realizes the type $p(x, y)$ then $(*)_{M, a, b}$ holds? Another variant is, given a model $M$ and $a, b \in M$ when do we have $(*)_{M, a, b}^{\text {pot }}$ or just $(*)_{M, a, b}^{\mathrm{tp}}$ ?

Our main aim is sorting this out. For the problem as stated, on the one hand we give a sufficient condition, and on the other hand, for $(*)_{M, a, b}^{\mathrm{tp}}$, we prove its necessity, assuming $\diamond_{\aleph_{1}}$. However, we may consider a relative:

Question 0.2 . Like question 0.1 but we restrict ourselves to $\aleph_{1}$-saturated models.

It seems natural to ask:

Date: May 22, 2012.

2010 Mathematics Subject Classification. Primary 03C62, 03C50; Secondary: 03C30, 03C55.

Key words and phrases. model theory, Peano arithmetic, linear order, automorphisms.

This research was supported by the Israel Science Foundation. Publication 924.

I would like to thank Alice Leonhardt for the beautiful typing. 
Question 0.3. Generally, how much does the linear order of a non-standard model $M$ of PA determine $M$ ? Is there a non-standard model $M$ of PA such that for every model $N$ of PA, if $(M \uparrow\{<\}) \cong(N, \uparrow\{<\})$, then are $M \cong N$ ?

We discussed those problems with Gregory Cherlin and he asked:

Question 0.4. [Cherlin] Show that $\{M \uparrow\{<\}: M \models \mathrm{PA}\}$ is complicated.

This question is too vague for my taste.

Recall the much earlier problem 14 from Kossak-Schmerl [KS06] asked by Friedman:

Question 0.5. Is there a model of PA such that for every model $N$ of PA, if $(M \uparrow\{<$ \}$) \cong(N, \uparrow\{<\})$, then $M \equiv N$ ?

This seems of different character, speaking just of the theory of another model, but of course, a positive answer to Question 0.3 would also give an answer to Question 0.5 .

We may go half way: maybe the linear order of $M$ does not determine $M$, say up to isomorphism, but just the additive structure (from which the order is definable). This means

Question 0.6. How much the order of $M$, a non-standard model of $T, T \in \mathrm{PA}^{\text {com }}$, determines the isomorphism type of $\left(|M|,<_{M},+_{M}\right)$ ?

A more general version of our question is

Question 0.7. Can we construct a non-standard model $M$ of PA with few order automorphisms in some sense?

Recall that for any countable non-standard model $M$ of PA, it is recursively saturated hence has "lots" of order automorphisms (see [KS06). Much has been done on other classes. Concerning Abelian groups and modules, see the book GöbelTrlifaj [GT06]. For general first order see [Sh:384] and history in both. In particular, there are non-standard models of PA with no automorphism, this motivating the "order-automorphism" in 0.7 Now answer to 0.1 sheds some light on 0.6 .

Let us review the present work. First, in $\$ 1$ we introduce and deal with some relevant equivalence relations, and in 1.8 it is proved that the so called $a E_{M}^{2} b$ implies $(*)_{M, a, b}$ so $a E_{M}^{2} b$ is a sufficient condition for a positive answer to Question 0.1(1), while for the so called 2-order rigid models $M$, we prove that the isomorphism type of $M \uparrow\{<\}$ determines that of $M \uparrow\{<,+\}$ but only up to almost isomorphism, shedding some light on 0.6 .

Second, in $\S 2$ we get that even $a E_{M}^{3} b$ implies $(*)_{M, a, b}$. This shows that 1.8 is not so interesting but its proof is a warm-up for $\S 2$. Moreover this is only part of the picture, see $\S 4$. In $\S 2$ we also show that if $M$ is 3 -o.r. then $M \uparrow\{<,+\}$ is unique up to almost isomorphism.

In $\S 3$ we show that $E_{M}^{3}$ is the right notion as if $\diamond_{\aleph_{1}}$ holds then every countable model of PA has elementary extension $M$ of cardinality $\aleph_{1}$ such that for $a, b \in M \backslash \mathbb{N}$ we have $a E_{M}^{3} b \Leftrightarrow M_{<a} \cong M_{<b}$. We comment there on the case $\neg a E_{M}^{4} b$.

Naturally, for most results some weaker version of PA suffices. We comment on this in $\S 4$; so usually when a result supercedes an earlier one, normally it has a harder proof and really use more axioms of PA.

We thank the referee for doing much to improve the presentation, considerably more than the call of duty and Shimoni Garti for help in proofreading. 
Convention 0.8. Models are models of PA, even ordinary ones if not said otherwise where $M$ is ordinary when $\mathbb{N} \subseteq M$. It is used e.g. in Definition 1.2(b),(c). We may circumvent it, defining $a+{ }_{M} n$ by repeated addition of $1_{M}$ and $a \times_{M} n$ by repeated additions of $a$, but it seems to me less convenient. 


\section{$\S 1$. SOMEWHAT RIGID ORDER}

We define (in 1.2) some equivalence relations $E_{M}^{\ell}$ for models $M$ (of PA). We shall deal with their basic properties in 1.5, 1.13, the relations between them (in 2.1), cofinalities of equivalence classes (in 1.12, 2.5), on order isomorphism/almost $\{<,+\}$-isomorphism in 1.1, 1.7, 2.4, including noting for $\ell$-o.r. models that $E_{M}^{\ell} \subseteq$ $E_{M}^{5}$.

Lastly, we prove versions of "if $M_{1} \uparrow\{<\}, M_{2} \uparrow\{<\}$ are isomorphic then $M_{1} \uparrow\{<$ $,+\}, M_{2} \uparrow\{<,+\}$ are almost isomorphic", see Theorems 1.8, 2.6.

Definition 1.1. We say $M, N$ are almost $\{<,+\}$-isomorphic when: some $f$ is an almost $\{<,+\}$-isomorphism from $M$ onto $N$ which means $f$ is an isomorphism from $M \uparrow\{<\}$ onto $N\lceil\{<\}$ such that for all $a, b \in M$ there is an $n \in \mathbb{N}$ such that the distance between $f\left(a+{ }_{M} b\right)$ and $f(a)+{ }_{N} f(b)$ is $n$.

Definition 1.2. We define the following equivalence relations on $M \backslash \mathbb{N}$

(a) $E_{M}^{0}: a E_{M}^{0} b$ iff $\bigvee_{n \in \mathbb{N}}\left(a<_{M} b+n \wedge b<_{M} a+n\right)$

(b) $E_{M}^{1}: a E_{M}^{1} b \underline{\text { iff }}$ for some $c \in M$ satisfying $n \in \mathbb{N} \Rightarrow n c<_{M} a, b$ we have $a<b+c$ and $b<a+c$

(c) $E_{M}^{2}: a E_{M}^{2} b$ iff $\bigvee_{n \in \mathbb{N}}\left(a<_{M} b \times_{M} n \wedge b<_{M} a \times_{M} n\right)$

(d) $E_{M}^{3}: a E_{M}^{3} b$ iff for some $c \in M$ we have $\bigwedge_{n \in \mathbb{N}}\left[c^{n}<_{M} a \wedge c^{n}<_{M} b\right.$ and $\left.a<b \times_{M} c \wedge b<a \times_{M} c\right]$

(e) $E_{M}^{4}: a E_{M}^{4} b$ iff $\bigvee_{n \in \mathbb{N}}\left(a<b^{n} \wedge b<a^{n}\right)$

(f) $E_{M}^{5}: a E_{M}^{5} b$ iff some order-automorphism of $M$ maps $a$ to $b$

(g) $E_{M}^{6}: a E_{M}^{6} b$ iff $a, b$ are equivalent for the minimal convex equivalent relations on $M$ which $E_{M}^{5}$ refines when

(h) an equivalence relation $E$ on a model $M=\left(|M|,<^{M}, \ldots\right)$ is convex when $a<_{M} b<_{M} c, a E c$ implies $a E b$.

Definition 1.3. For $\ell \in\{0,1, \ldots, 6\}, M$ is called $\ell$-o.r. (for order-rigid) if for all $a, b \in M,\left(M_{<a},<_{M}\right) \cong\left(M_{<b},<_{M}\right)$ implies that $a E_{M}^{\ell} b$.

Discussion 1.4. While we know that there are rigid linear orders and we know that there are rigid models of PA, it is harder to build $\ell$-.o.r. models of PA, our relevant result will be Theorem 3.10 .

Claim 1.5. 1) For $\ell \in\{0, \ldots, 6\}$ the two place relation $E_{M}^{\ell}$ is an equivalence relation on $M \backslash \mathbb{N}$ and is convex except possibly for $\ell=5$.

1A) Moreover, if $a E_{M}^{3} b \underline{\text { then }}$ for every $c \in M$ we have $M \models\left(\bigwedge_{n} c^{n}<a\right) \Leftrightarrow M \models$ $\left(\bigwedge_{n} c^{n}<b\right)$ and the set of such $c^{\prime}$ s is closed under products and sums and is convex. 1B) Also if a $E_{M}^{1} b \underline{\text { then }}$ for every $c \in M$ we have $M \models$ "( $\left.\bigwedge_{n} c \times n<a\right) \Leftrightarrow\left(\bigwedge_{n}(c \times n<\right.$ b)" and the set of such c's is closed under sums and is convex.

2) If $a, b \in M \backslash \mathbb{N}$ and $a E_{M}^{2} b$ then $\left(M_{<a},<_{M}\right) \cong\left(M_{<b},<_{M}\right)$; moreover there is an automorphism of $M \uparrow\{<\}$ mapping a to b, that is, $a E_{M}^{5} b$.

3) Assume $a, b \in M \backslash \mathbb{N} \underline{\text { then }}\left(M_{<a},<_{M}\right) \cong\left(M_{<b},<_{M}\right)$ iff $a E_{M}^{5} b$. 
4) $a E_{M}^{6} b$ iff there is $c \leq_{M} \min \{a, b\}$ and an order-automorphism $f$ of $M$ such that $\max \{a, b\} \leq_{M} f(c)$ iff this holds for $c=\min \{a, b\}$.

Remark 1.6. Note that in $\S 2$ we establish a stronger version of 1.5(2), see 2.4 but the proof of 2.4 uses 1.5(2), also the proof of 1.5(2) applies to weaker versions of PA than the proof of 2.4, see $\S 4$.

Proof. 1) Let $\ell=3$. If $a_{1} E_{M}^{3} a_{2}$ and let $c$ witness it, then $c$ witnesses also $a_{2} E_{M}^{3} a_{2}$ and $a_{2} E_{M}^{3} a_{1}$, so reflexivity and symmetry holds.

Lastly, assume $M \models " a_{1}<a_{2}<a_{3}$ "; if $a_{k} E_{M}^{3} a_{k+1}$ and let $c_{k}$ witness this for $k=1,2$, then the product $c_{1} c_{2}$ witness $a_{1} E_{M}^{3} a_{3}$ by part (1A) proved below and if $a_{1} E_{M}^{3} a_{3}$ then the same witness gives $a_{1} E_{M}^{3} a_{2} \wedge a_{2} E_{M}^{3} a_{3}$ so transitivity and convexity holds.

For $\ell=1$ the proof is similar (using part (1B) instead of part (1A)), also for $\ell=0,2,4$ the proof is even easier and for $\ell=5,6$ it holds by the definition.

1A), 1B) Check.

2) Without loss of generality, assume that $a<(n-1) a<b<n a$, where $1 \leq n \in \mathbb{N}$, and also there is a $c$ such that $(n-1) c=n a-b$. Then $c<a$ because otherwise $(n-1) a \leq(n-1) c=n a-b$ hence $b \leq a$, contradiction. Let $X$ be a set of representatives for $M / E_{0}$ and, without loss of generality, assume $a, c \in X$. Now define $f: M \rightarrow M$ by first defining it on $X$ and then extending it to all of $M$ in the obvious way. (The obvious way is: if $y=x+k$, where $x \in X$ and $k \in \mathbb{Z}$, then $f(y)=f(x)+k$ and $f$ is the identity on $\mathbb{N}$.) If $x \in X$, then let

$$
f(x)= \begin{cases}x & \text { if } x \leq c, \\ n(x-a)+b & \text { otherwise. }\end{cases}
$$

Clearly, $f(c)=c$ and $f(a)=b$. Now check that $f$ is as required.

3) First, if $f$ exemplifies $a E_{M}^{5} b$, i.e. is an automorphism of $M$ mapping $a$ to $b$ then $f \uparrow M_{<a}$ is an isomorphism from $\left(M_{<a},<_{M}\right)$ onto $\left(M_{<b},<_{M}\right)$. Second, if $f$ is an isomorphism from $\left(M_{<a},<_{M}\right)$, onto $\left(M_{<b},<_{M}\right)$ we define a function $g: M \rightarrow M$ by: $g(c)$ is $f(c)$ if $c<_{M} a$ and is $b+(c-a)$ if $a \leq_{M} c$. Now check.

4) Let $E_{M}^{\prime}=\{(a, b)$ : for some order-automorphism $f$ of $M$ we have $f(\min \{a, b\}) \geq$ $\max \{a, b\}\}$; clearly this is symmetric (by definition), reflexive (using $f=$ the identity) and as $f$ is monotonic also convex (i.e. $a \leq a_{1} \leq b_{1} \leq b \wedge a E_{M}^{\prime} b \Rightarrow$ $\left.a_{1} E_{M}^{\prime} b_{1}\right)$. To prove transitivity it is now enough to show $a_{1}<a_{2}<a_{3} \wedge a_{1} E_{M}^{\prime} a_{2} \wedge$ $a_{2} E_{M}^{\prime} a_{3} \Rightarrow a_{1} E_{M}^{\prime} a_{3}$ which hold by composing the automorphisms $f_{1}, f_{2}$ witnessing $a_{1} E_{M}^{\prime} a_{2}, a_{2} E_{M}^{\prime} a_{3}$ respectively. So $E_{M}^{\prime}$ is a convex equivalence relation and obviously $a E_{M}^{5} b \Rightarrow a E_{M}^{\prime} b$.

Lastly, $E_{M}^{\prime}$ is refined by any convex equivalence relation refining $E_{M}^{5}$, so it follows that $E_{M}^{6}=E_{M}^{\prime}$ so we are done.

Claim 1.7. 1) If $f$ is an order-isomorphism from $M_{1}$ onto $M_{2}$ then $f$ maps $E_{M_{1}}^{0}$ onto $E_{M_{2}}^{0}$.

2) If $f$ is an almost $\{<,+\}$-isomorphism from $M_{1}$ onto $M_{2}$ then $f$ maps $E_{M_{1}}^{1}$ onto $E_{M_{2}}^{1}$ and $E_{M_{1}}^{2}$ onto $E_{M_{2}}^{2}$.

3) Similarly for embeddings (not used).

Proof. Straight.

Recalling Definition 1.3(2) 
Theorem 1.8. If $M_{1}$ is 2-o.r. and $M_{1} \uparrow\{<\}, M_{2} \uparrow\{<\}$ are isomorphic then $M_{1}, M_{2}$ are almost $\{<,+\}$-isomorphic.

Remark 1.9. 1) We have not said "by the same isomorphism".

2) The assumption is too strong to be true for models of full PA. But it makes sense for weaker versions of PA, see 4.5 and part of the proof serves as proof to 2.7 so indirectly serves 2.6 ,

Question 1.10. Are $M_{1}, M_{2}$ isomorphic when (the main case is $\ell=3$ ):

(a) $M_{1}, M_{2}$ are isomorphic as linear orders

(b) $M_{1}$ is $\ell$-o.r.

Remark 1.11. In 1.10, it is less desirable but we may consider adding that also $M_{2}$ is l.o.r.

Proof. Easily by $1.5(1)$

$(*)_{0}$ each $E_{M_{\ell}}^{2}$-equivalence class is convex.

Without loss of generality

$(*)_{1}<M_{1}=<_{M_{2}}$ hence $M_{1}, M_{2}$ have the same universe and let $M:=M_{1} \uparrow\{<\}=$ $M_{2} \uparrow\{<\}$.

Also as usual

$(*)_{2} \quad \mathbb{N} \subseteq M_{\ell}$ for $\ell=1,2$.

Now

$(*)_{3}$ if $a, b \in M \backslash \mathbb{N}$ and $a<_{M} b$ and $a+{ }_{M_{2}} b=c$ and $a^{\prime}=c--_{M_{1}} b$, that is $M_{1} \models$ " $c=a^{\prime}+b$ " then $a E_{M_{1}}^{2} a^{\prime}$.

[Why? Now $[b, c)_{M_{2}}=[b, c)_{M}$ is isomorphic to $[0, a)_{M_{2}}$ as linear orders (as $M_{2} \models$ " $a+b=c "$ as $M_{2}$ satisfies PA) hence $[b, c)_{M_{1}}$ is isomorphic to $[0, a)_{M_{1}}$ as linear orders. Of course $[b, c)_{M_{1}}$ is isomorphic to $\left[0, c-M_{1} b\right)=\left[0, a^{\prime}\right)_{M_{1}}$ as linear orders. So $[0, a)_{M_{1}},\left[0, a^{\prime}\right)_{M_{1}}$ are isomorphic as linear orders. But $M_{1}$ is 2-o.r. hence $a E_{M_{1}}^{2} a^{\prime}$ as required].

$(*)_{4}$ if $a<_{M} b$ and $b \in M \backslash \mathbb{N}$ then $b, a+{ }_{M_{1}} b, a+_{M_{2}} b$ are $E_{M_{1}}^{2}$-equivalent.

[Why? Similar proof: as by the proof of $1.5(3)$ as trivially $b E_{M_{\ell}}^{2}\left(a+M_{\ell} b\right)$ for $\ell=1,2$ and $\left(a+{ }_{M_{1}} b\right) E_{M_{1}}^{5}\left(a+M_{2} b\right)$ so use " $M_{1}$ is 2-o.r." to deduce $\left(a+{ }_{M_{1}} b\right) E_{M_{1}}^{2}\left(a+{ }_{M_{2}} b\right)$, together we are done.]

$(*)_{5}$ if $a, b \in M \backslash \mathbb{N}$ and $a \times_{M_{\ell}} b=c_{\ell}$ for $\ell=1,2$ then $c_{1} E_{M_{1}}^{2} c_{2}$.

[Why? For $\ell=1,2$, as $M_{\ell}$ is a model of PA it follows that $\left(M_{<c_{\ell}},<_{M}\right)$ is isomorphic to $\left(M_{<a},<_{M}\right) \times\left(M_{<b},<_{M}\right)$ ordered lexicographically. Hence $\left(\left(M_{1}\right)_{<c_{1}}\right.$, $\left.<_{M}\right)=\left(M_{<c_{1}},<_{M}\right)$ and $\left(\left(M_{2}\right)_{<c_{2}},<_{M}\right)=\left(M_{<c_{2}},<_{M}\right)$ are isomorphic (and trivially $c_{1}, c_{2} \notin \mathbb{N}$ ) hence by " $M_{1}$ is 2-o.r." we have $c_{1} E_{M_{1}}^{2} c_{2}$ as promised.]

$(*)_{6}$ for $a, b \in M \backslash \mathbb{N}$ we have:

$$
a E_{M_{2}}^{2} b \text { iff } a E_{M_{1}}^{2} b \text {. }
$$


[Why? First, assume $a E_{M_{2}}^{2} b$; now without loss of generality $a<_{M} b$, and say $k \in \mathbb{N}, k \neq 0, b<k \times_{M_{2}} a$ so $b<a+_{M_{2}} \ldots+_{M_{2}} a$ ( $k$ summands). By $(*)_{4}$ we can prove by induction on $k$ that $b E_{M_{1}}^{2} a$ as required in the "only if" direction. (Alternatively by $1.5(2)$ we have $a E_{M}^{5} b$ hence by " $M_{1}$ is 2-o.r.", we get $b E_{M_{1}}^{2} a$.)

Second, assume $\neg\left(a E_{M_{2}}^{2} b\right)$ and without loss of generality $a<_{M} b$; note that we cannot use the same argument as above interchanging $M_{1}, M_{2}$, because only on $M_{1}$ we assume its being 2 - o.r. As $M_{2} \models$ PA there is $c \in M_{2}$ such that $M_{2} \models " a \times c \leq b<a \times c+a$ ", now $c \notin \mathbb{N}$ because we are assuming $\neg\left(a E_{M_{2}}^{2} b\right)$. By $(*)_{5}$ we have $\left(a \times_{M_{1}} c\right) E_{M_{1}}^{2}\left(a \times_{M_{2}} c\right)$. But by $(*)_{4}$, we have $a \times_{M_{2}} c, a \times_{M_{2}} c+_{M_{2}} a$ are $E_{M_{1}}^{2}$-equivalent hence by the choice of $c$ and $(*)_{0}$ also $a \times_{M_{2}} c, b$ are $E_{M_{1}}^{2}$ equivalent; so together with the previous sentence $\left(a \times_{M_{1}} c\right) E_{M_{1}}^{2} b$. But by the definitions, as $c \in M \backslash \mathbb{N}$ clearly $\neg\left(a E_{M_{1}}^{2}\left(a \times_{M_{1}} c\right)\right)$ hence $\neg\left(a E_{M_{1}}^{2} b\right)$ as required in the "if" direction.]

$(*)_{7}$ if $a \in M \backslash \mathbb{N}$ then $\left(a / E_{M_{1}}^{2},<_{M}\right)$ has cofinality $\aleph_{0}$ and also its inverse has cofinality $\aleph_{0}$.

[Why? As $M_{1} \models \mathrm{PA}$ the sequence $\left\langle a \times_{M_{1}} 2^{n}: n \in \mathbb{N}\right\rangle$ is increasing, the members form an unbounded subset of $a / E_{M_{1}}^{2}$; similarly $\left\langle\min \left\{b \in M: a \leq b \times_{M_{1}} 2^{n}\right\}: n \in \mathbb{N}\right\rangle$ is decreasing, the members form a subset of $a / E_{M_{1}}^{2}$ unbounded from below, recalling the definition of $E_{M_{1}}^{2}$.]

$(*)_{8}$ let $\ell \in\{1,2\}$ if $a \in M \backslash \mathbb{N}$ and $X_{\ell}=\left\{\left(2^{b}\right)^{M_{\ell}}: b \in M_{\ell}\right\}$ for $\ell=1,2$ then $X_{\ell} \cap\left(a / E_{M_{1}}^{2}\right)$ has order-type $\mathbb{Z}$ and is unbounded in $\left(a / E_{M_{1}}^{2},<_{M}\right)$ from above and from below.

[Why? If $a^{\prime} \in M \backslash \mathbb{N}$ and $\ell \in\{1,2\}$, then as $M_{\ell} \models \mathrm{PA}$ for some $b_{\ell}$ we have $M_{\ell}=" 2^{b_{\ell}} \leq a^{\prime}<2^{b_{\ell}+1}=2^{b_{\ell}}+2^{b_{\ell}}$ " so recalling the definition of $E_{M_{\ell}}^{2}$ we are done.]

We may hope that: if $a \in M \backslash \mathbb{N}$ and $M_{\ell} \models$ " $2^{a}=b_{\ell}$ " for $\ell=1,2$ then $b_{1} E_{M_{1}}^{2} b_{2}$. Anyhow

$\circledast_{1}$ for $\ell=1,2$

(a) define $f_{\ell}: M_{\ell} \rightarrow M_{\ell}$ by $f_{\ell}(a)=\left(2^{a}\right)^{M_{\ell}}$,

(b) define: $M_{\ell}^{*}$ is the model with universe $X_{\ell}:=\operatorname{Rang}\left(f_{\ell}\right)$ such that $f_{\ell}$ is an isomorphism from $M_{\ell}$ onto $M_{\ell}^{*}$

$\circledast_{2}$ for $\ell=1,2$ if $a, b \in X_{\ell}$ then $a+{ }_{M_{\ell}^{*}} b=a \times_{M_{\ell}} b$.

[Why? As PA $\vdash 2^{x} 2^{y}=2^{x+y}$.]

$\circledast_{3}$ if $\ell=1,2$ and $a, b \in M_{1} \backslash \mathbb{N}$ then

(a) $a E_{M_{\ell}}^{0} b$ iff $f_{\ell}(a) E_{M_{\ell}^{*}}^{2} f_{\ell}(b)$

(b) $a E_{M_{\ell}}^{1} b$ iff $f_{\ell}(a) E_{M_{\ell}^{*}}^{3} f_{\ell}(b)$

(c) $a E_{M_{\ell}}^{2} b$ iff $f_{\ell}(a) E_{M_{\ell}^{*}}^{4} f_{\ell}(b)$.

[Why? Look at the definitions and do basic arithmetic.]

$\circledast_{4}$ there is an order isomorphism $h$ from $X_{1}$ onto $X_{2}$ such that

(a) $h\left\lceil\left\{\left(2^{n}\right)^{\mathbb{N}}: n \in \mathbb{N}\right\}\right.$ is the identity

(b) if $a \in M \backslash \mathbb{N}$ then $h$ maps $X_{1} \cap\left(a / E_{M_{1}}^{2}\right)$ onto $X_{2} \cap\left(a / E_{M_{2}}^{2}\right)$. 
[Why? By $(*)_{8}+(*)_{6}$.]

$\circledast_{5}$ if $a, b \in X_{1}$ then $a E_{M_{1}^{*}}^{0} b \Leftrightarrow h(a) E_{M_{2}^{*}}^{0} h(b)$.

[Why? By $\circledast_{3}$ and $\circledast_{4}$ and the definition of $E_{M_{\ell}^{*}}^{0}$ ]

$\circledast_{6}$ if $M_{\ell}^{*} \models " a_{\ell}+b_{\ell}=c_{\ell}$ " for $\ell=1,2$ and $h\left(a_{1}\right)=a_{2}$ and $h\left(b_{1}\right)=b_{2}$ then

(a) $c_{1} E_{M_{\ell}}^{2} c_{2}$ for $\ell=1,2$

(b) $c_{1} E_{M_{\ell}^{*}}^{0} c_{2}$ for $\ell=1,2$.

[Why? If $a_{1} \in \mathbb{N}$ or $b_{1} \in \mathbb{N}$ the conclusion follows easily so we assume $a_{1}, b_{1} \notin \mathbb{N}$. For $\ell=1,2$ by $\circledast_{2}$ we have $M_{\ell} \models$ " $a_{\ell} \times b_{\ell}=c_{\ell}$ ". Also by $\circledast_{4}(b)$ we have $x \in X_{1} \backslash \mathbb{N} \Rightarrow$ $x E_{M_{1}}^{2} h(x)$ recalling $E_{M_{1}}^{2}=E_{M_{2}}^{2}$ by $(*)_{6}$ we have $a_{1} E_{M_{2}}^{2} a_{2}, b_{1} E_{M_{2}}^{2} b_{2}$ hence for some $n \in \mathbb{N}$ we have $M_{2} \models$ " $a_{1}<n \times a_{2} \wedge a_{2}<n \times a_{1} \wedge b_{1}<n \times b_{2} \wedge b_{2}<n \times b_{1}$ " hence $M_{2} \models$ " $a_{1} \times b_{1}<n^{2} \times a_{2} \times b_{2} \wedge a_{2} \times b_{2}<n^{2} \times a_{1} \times b_{1}$ " hence $\left(a_{1} \times_{M_{2}} b_{1}\right),\left(a_{2} \times_{M_{2}} b_{2}\right)$ are $E_{M_{2}}^{2}$-equivalent and also are $E_{M_{2}}^{2}$-equivalent.

So by $(*)_{5}$ we have $\left(a_{1} \times_{M_{2}} b_{1}\right),\left(a_{1} \times_{M_{1}} b_{1}\right)$ are $E_{M_{1}}^{2}$-equivalent and also $\left(a_{2} \times_{M_{1}}\right.$ $\left.b_{2}\right),\left(a_{2} \times_{M_{2}} b_{2}\right)$ are $E_{M_{1}}^{2}$-equivalent hence together with the previous paragraph by $(*)_{6}$ they are $E_{M_{\ell}}^{2}$-equivalent, in particular $c_{1}, c_{2}$ are $E_{M_{\ell}}^{2}$-equivalent as required in clause (a) of $\circledast_{6}$. By $\circledast_{3}+\circledast_{1}(b)$ also clause (b) of $\circledast_{6}$ there follows.]

So by $\circledast_{4}+\circledast_{6}(b)$ we are done.

We have used

Observation 1.12. Assume $a \in M \backslash \mathbb{N}$

1) $\langle a+n: n \in \mathbb{N}\rangle$ is increasing and cofinal in $a / E_{M}^{0}$.

2) $\langle a-n: n \in \mathbb{N}\rangle$ is decreasing and unbounded from below in $a / E_{M}^{0}$.

3) $\langle n \times a: n \in \mathbb{N}\rangle$ is increasing and cofinal in $a / E_{M}^{2}$.

4) Moreover $\left.\left\langle\min \left\{b: n \times_{M} b \geq a\right\}: n \in \mathbb{N}\right\}\right\rangle$ is decreasing and unbounded from below in $a / E_{M}^{2}$.

5) Moreover for some $b, 2^{b} \leq a<2^{b+1}$ hence we can use in (3),(4) the sequence $\left\langle 2^{b+n}: n \in \mathbb{N}\right\rangle,\left\langle 2^{b-n}: n \in \mathbb{N}\right\rangle$.

Observation 1.13. 1) Assume $M_{k} \models$ " $a \times b=c_{k}$ " for $k=1,2$ and $M_{1} \uparrow\{<\}=$ $M_{2} \uparrow\{<\}$. Then $c_{1} E_{M_{2}}^{5} c_{2}$ for $k=1,2$.

2) Assume $M_{k} \models$ " $a_{1} \times a_{2} \times \ldots \times a_{m}=c_{k}$ " for $k=1,2$ and $M_{1} \uparrow\{<\}=M_{2} \uparrow\{<\}$. Then $c_{1} E_{M_{k}}^{5} c_{2}$ for $k=1,2$.

Proof. 1) See $(*)_{5}$ in the proof of 1.8

2) Similar proof. 


\section{$\S 2$. MORE FOR $E_{M}^{3}$}

Here we say more on the equivalence relations $E_{M}^{\ell}$. In 2.1 we deal with basic properties: when $E_{\mu}^{\ell} \subseteq E_{\mu}^{\ell+1}$, when $\ell$-o.r. implies $(\ell+1)$-o.r., preservation under + and $\times$. We also prove one half of our answer to 0.1 that is in 2.4 we prove $a_{1} E_{M}^{3} b$ implies $a_{1} E_{\mu}^{5} a_{2}$. Concerning the weak form of uniqueness of the additive structure in Theorem 2.6 we prove e.g. if $M_{1}, M_{2}$ are order isomorphic and $M_{1}$ is 3-o.r. then $M_{1}\left\lceil\{<,+\}, M_{2} \uparrow\{<,+\}\right.$ are almost isomorphic (i.e. the "error" in + is finite) but not necessarily by the same isomorphism. We end (in 2.9) that $a / E_{\mu}^{4}$ is divided by $E_{\mu}^{3}$ if $I=\left\{b / E_{\mu}^{3}: b \in a / E_{\mu}^{4}\right\}$ is naturally ordered, is isomorphic to a subset of $\mathbb{R}$, even one which is an additive subgroup (a "translation" of the product in $M$ ).

Claim 2.1. 1) $E_{M}^{\ell}$ refines $E_{M}^{\ell+1}$ for $\ell=0,1,2,3,5$.

2) If $a_{k} E_{M}^{\ell} b_{k}$ for $k=1,2$ and $\ell=0,1,2,3,4,5,6 \underline{\text { then }}\left(a_{1}+a_{2}\right) E_{M}^{\ell}\left(b_{1}+b_{2}\right)$.

3) If $a_{k} E_{M}^{\ell} b_{k}$ for $k=1,2$ and $\ell=2,3,4$ then $\left(a_{1} \times{ }_{M} a_{2}\right) E_{M}^{\ell}\left(b_{1} \times_{M} b_{2}\right)$.

4) Part (3) holds also for $\ell=5,6$.

5) If $M$ is $\ell$-o.r. then $M$ is $(\ell+1)$-o.r. for $\ell=0,1,2,3$.

Remark 2.2. Concerning 2.1] recall that $E_{M}^{3}$ refines $E_{M}^{5}$ by [1.5)(3),(4).

Proof. 1) Read the definitions.

2) First, assume $\ell=0$, so by the assumption for $k=1,2$ there are $m_{k}, n_{k} \in \mathbb{N}$ such that $M \models$ " $a_{k}+m_{k}=b_{k}+n_{k}$ ". Now let $m:=m_{1}+m_{2} \in \mathbb{N}$ and $n:=n_{1}+n_{2} \in \mathbb{N}$ hence $M \models$ " $\left(a_{1}+a_{2}\right)+\left(m_{1}+m_{2}\right)=\left(b_{1}+b_{2}\right)+\left(n_{1}+n_{2}\right)$ " hence $\left(a_{1}+a_{2}\right) E_{M}^{0}\left(b_{1}+b_{2}\right)$ as required.

Second, assume $\ell=2$, so by the assumption, for $k=1,2$ there is $n_{k} \in \mathbb{N}$ such that $M \models " a_{k}<n_{k} \times b_{k} \wedge b_{k}<n_{k} \times a_{k}$ ". Let $n=\max \left\{n_{1}, n_{2}\right\} \in \mathbb{N}$ hence $M \models$ " $\left(a_{1}+a_{2}\right)<n_{1} b_{1}+n_{2} b_{2} \leq n b_{1}+n b_{2}=n\left(b_{1}+b_{2}\right)$ " and similarly $M \models "\left(b_{1}+b_{2}\right)<n\left(a_{1}+a_{2}\right)$ " hence $\left(a_{1}+a_{2}\right) E_{M}^{2}\left(b_{1}+b_{2}\right)$.

Third, assume $\ell=1,3$; without loss of generality $a_{1}<_{M} b_{1}$ and as $E_{M}^{\ell}$ is convex (see 1.5(1)) without loss of generality $a_{2} \leq_{M} b_{2}$. Letting $c_{k}$ witness $a_{k} E_{M}^{\ell} b_{k}$ for $k=1,2$ easily $c=\max \left\{c_{1}, c_{2}\right\}$ witness $\left(a_{1}+a_{2}\right) E_{M}^{\ell}\left(b_{1}+b_{2}\right)$.

Fourth, the case $\ell=4$ is easy, too.

Fifth, assume $\ell=5$ and $f_{k}$ is an order-automorphism of $M$ mapping $a_{k}$ to $b_{k}$ for $k=1,2$. Define a function $f$ from $M$ to $M$ by

$(*) \quad(a) \quad f(x)=f_{1}(x)$ if $x<_{M} a_{1}$

$$
\text { (b) } f(x)=b_{1}+f_{2}\left(x-a_{1}\right) \text { if } a_{1} \leq_{M} x .
$$

Now check.

Sixth, assume $\ell=6$, let $c_{k}=\min \left\{a_{k}, b_{k}\right\}$; by 1.5)(4) there is $d_{k} \geq \max \left\{a_{k}, b_{k}\right\}$ such that $c_{k} E_{M}^{5} d_{k}$ so $a_{k}, b_{k} \in\left[c_{k}, d_{k}\right]$ for $k=1,2$. But $\left(c_{1}+c_{2}\right) E_{M}^{5}\left(d_{1}+d_{2}\right)$ by the result for $\ell=5$ and $a_{1}+b_{1}, a_{2}+b_{2} \in\left[c_{1}+c_{2}, d_{1}+d_{2}\right]$ so we are done.

3) First, assume $\ell=2$ and for $k=1,2$ let $n_{k}$ witness $a_{k} E_{M}^{2} b_{k}$ and choose $n=n_{1} n_{2}$ noting that $n_{1}, n_{2}>0$ by Definition 1.2 (c). Now $M \models " a_{1} \times a_{2}<\left(n_{1} \times b_{1}\right) \times\left(n_{2} \times\right.$ $\left.b_{2}\right)=n \times\left(b_{1} \times b_{2}\right)$ " and similarly $M \models\left(b_{1} \times b_{2}\right)<n\left(a_{1} \times a_{2}\right)$.

The proof for $\ell=3$ is easy, too. For $\ell=4$ by the convexity of $E_{M}^{4}$ without loss of generality $a_{1} \leq a_{2}, b_{1} \leq b_{2}$ and so there are $n, m \in \mathbb{N}$ such that $a_{2} \leq a_{1}^{n}, b_{2} \leq b_{1}^{m}$, so $a_{1} \times b_{1} \leq a_{2} \times b_{2} \leq a_{1}^{n} \times b_{1}^{m} \leq\left(a_{1} \times b_{1}\right)^{n+m}$ hence $\left(a_{1} \times b_{1}\right) E_{M}^{4}\left(a_{2} \times b_{2}\right)$.

4) For $\ell=5$, as in the proof of 1.8 , i.e. if $c_{k}=a_{k} \times{ }_{M} b_{k}$ for $k=1,2$ there is an order isomorphism $h_{k}$ from $M_{<a_{k}} \times M_{<b_{k}}$ onto $M_{<c_{k}}$ and let $f_{k}$ be an order automorphism 
of $M$ mapping $a_{k}$ to $b_{k}$. Combining there is an order-isomorphism $g_{1}$ from $M_{<c_{1}}$ onto $M_{<c_{2}}$ and let $g$ be the order automorphism of $M$ such that $g$ extends $g_{1}$ and $c_{1} \leq d \in M \Rightarrow g(d)=c_{2}+f_{1}\left(d-c_{1}\right)$; so $g$ witness $\left(a_{1} \times b_{1}\right) E_{M}^{5}\left(a_{2} \times b_{2}\right)$ as promised.

For $\ell=6$ it follows in the proof of part (3).

$5)$ By the definition of $m$-o.r. and part (1).

Question 2.3. Is $E_{M}^{5}$ convex for every $M$ ?

Claim 2.4. If $a_{1} E_{M}^{3} a_{2}$ then there is an order-automorphism of $M$ mapping $a_{1}$ to $a_{2}$, i.e. $a_{1} E_{M}^{5} a_{2}$.

Proof. Without loss of generality $a_{1}<_{M} a_{2}$. If $a_{1} E_{M}^{2} a_{2}$ then $a_{1} E_{M}^{5} a_{2}$ by [1.5 (2), so without loss of generality $\neg\left(a_{1} E_{M}^{2} a_{2}\right)$ hence $n \times a_{1}<a_{2}$ for $n \in \mathbb{N}$. So by the definition of $E_{M}^{3}$ and the assumption $a_{1} E_{M}^{3} a_{2}$, clearly for some $c \in M \backslash \mathbb{N}$ we have

$(*)_{1} \quad c<_{M} a_{1}, M \models " c c^{n}<a_{1}$ " for $n \in \mathbb{N}$ and $(c-1) \times_{M} a_{1}<_{M} a_{2} \leq_{M} c \times_{M} a_{1}$.

Clearly $a_{2} E_{M}^{2}\left(c \times_{M} a_{1}\right)$ hence again by $1.5(2)$ without loss of generality

$(*)_{2} \quad M \models " a_{2}=c \times_{M} a_{1} "$.

We define an equivalence relation $E$ on $M \backslash \mathbb{N}$ :

$(*)_{3} x E y$ iff $\bigvee_{n}|x-y|<c^{n}$.

Clearly $E$ is a convex equivalence relation. We choose a set $X$ of representatives for $E$ such that $0, a_{1}, a_{2} \in X$, can be done as $0+c^{n}=c^{n}<a_{1}$ and $c^{n}+a_{1}<2 \times a_{1}<a_{2}$ for any $n \in \mathbb{N}$.

Note

$(*)_{4}$ if $b_{1}, b_{2} \in M_{\leq a_{1}}$ then $\left(b_{1} E b_{2}\right) \Leftrightarrow\left(c \times b_{1}\right) E\left(c \times b_{2}\right)$.

[Why? As we have

$$
\left|\left(c \times b_{2}\right)-\left(c \times b_{1}\right)\right|=c \times\left(\left|b_{2}-b_{1}\right|\right)
$$

so for $n \in \mathbb{N}$ :

$$
\left|\left(c \times b_{2}\right)-\left(c \times b_{1}\right)\right|<c^{n+1} \Leftrightarrow\left|b_{2}-b_{1}\right|<c^{n}
$$

so $(*)_{4}$ is true indeed.]

Now we define a function $f$ from $M$ into $M$ as follows:

$(*)_{5}(a) \quad$ if $x \in 0 / E$, i.e. $\bigvee_{n} x<_{M} c^{n}$ then $f(x)=x$

(b) if $y \in X$ and $y \neq 0$ then $f(y)=c \times y$

(c) if $x \in X \backslash(0 / E) \wedge x \leq_{M} y \in x / E$ then $f(y)=f(x)+(y-x)$

(d) $\quad$ if $x \in X \backslash(0 / E) \wedge y<_{M} x \wedge y \in x / E$ then $f(y)=f(x)+(x-y)$

Note that $f$ is well defined and is one-to-one order preserving and onto $M$ by $(*)_{4}$. As $f\left(a_{2}\right)=c \times a_{1}=a_{2}$ we have $a_{1} E a_{2}$ so we are done.

प2.4

Comparing with $(*)_{7}$ of the proof of 1.8

Observation 2.5. 1) For any $a \in M \backslash \mathbb{N}$ we have: 
(a) the sequence $\left\langle\left\lfloor a^{1+2^{-n}}\right\rfloor: n \in \mathbb{N}\right\rangle$, that is $\langle\max \{b: b$ in $M, a$ divides $b$ and $\left.\left.(\lfloor b / a\rfloor)^{2^{n}} \leq a\right\}: n \in \mathbb{N}\right\rangle$ is a decreasing sequence from $\left\{b: a^{\prime}<b\right.$ for every $\left.a^{\prime} \in a / E_{M}^{3}\right\}$ unbounded from below in it

(b) the sequence $\left\langle\left\lfloor a^{1-2^{-n}}\right\rfloor: n \in \mathbb{N}\right\rangle$, that is $\left\langle\max \left\{b:(\lfloor a / b\rfloor)^{2^{n}} \leq a\right\}: n \in \mathbb{N}\right\rangle$ is an increasing sequence included in $\left\{b: b<a^{\prime}\right.$ for every $\left.a^{\prime} \in a / E_{M}^{3}\right\}$ and unbounded from above in it.

2) For $a \in M \backslash \mathbb{N}$ we have:

(a) the sequence $\left\langle\left\lfloor\left(1+2^{-n}\right) a\right\rfloor: n \in \mathbb{N}\right\rangle$, is a decreasing sequence in $\{b \in M: b$ above $\left.a / E_{M}^{1}\right\}$ cofinal in it

(b) the sequence $\left\langle\left\lfloor\left(1+2^{-n}\right) a\right\rfloor: n \in \mathbb{N}\right\rangle$, is an increasing sequence in $\{b \in M: b$ below $\left.a / E_{M}^{1}\right\}$ cofinal in it.

Proof. Straight.

Theorem 2.6. 1) If $M_{1}$ is 3-o.r. and $f$ is an order-isomorphism from $M_{1}$ onto $M_{2}$ then $f$ maps $E_{M_{1}}^{k}$ onto $E_{M_{2}}^{k}$ for $k=3,4$.

2) In part (1), moreover $M_{1} \uparrow\{<,+\}, M_{2} \uparrow\{<,+\}$ are almost isomorphic.

3) For any $M$ let $E_{M}^{7}=\left\{(a, b):\left(\left\lfloor\log _{2}(a)\right\rfloor\right) E_{M_{1}}^{4}\left(\left\lfloor\log _{2}(b)\right\rfloor\right)\right\}$. Assume there is an order-isomorphism $f$ from $M_{1}$ onto $M_{2}$ mapping $E_{M_{1}}^{4}$ to $E_{M_{2}}^{4}$, e.g. as in the conclusion of part (1) and $f$ maps $E_{M_{1}}^{7}$ onto $E_{M_{2}}^{7}$ then $M_{1} \uparrow\{<,+\}, M_{2} \uparrow\{<,+\}$ are almost $\{<,+\}$ isomorphic.

Proof. 1) By the assumption and by 2.4 respectively

$(*)_{0} \quad(a) \quad E_{M_{1}}^{3} \supseteq E_{M_{1}}^{5}$

(b) $E_{M_{\ell}}^{3} \subseteq E_{M_{\ell}}^{5}$ for $\ell=1,2$.

Easily by $1.5(1)$

$(*)_{1}$ each $E_{M_{\ell}}^{3}$-equivalence class is convex.

Without loss of generality

$(*)_{2} \quad(a) \quad<_{M_{1}}=<_{M_{2}}$ hence

(b) $M_{1}, M_{2}$ have the same universe and

(c) $E_{M_{1}}^{5}=E_{M_{2}}^{5}$ so $E_{M_{2}}^{3} \subseteq E_{M_{2}}^{5} \subseteq E_{M_{1}}^{5}=E_{M_{1}}^{3}$

(d) let $M:=M_{1} \uparrow\{<\}=M_{2} \uparrow\{<\}$ hence $E_{M_{1}}^{5}=E_{M}^{5}=E_{M_{2}}^{5}=E_{M_{1}}^{3}$.

Also as usual

$(*)_{3} \mathbb{N} \subseteq M_{k}$ for $k=1,2$.

$(*)_{4}$ if $a<_{M} b$ and $b \in M \backslash \mathbb{N}$ then $b, a+{ }_{M_{1}} b, a+{ }_{M_{2}} b$ are $E_{M_{1}}^{3}$-equivalent.

[Why? By the definition of $E_{M}^{3}$.]

$(*)_{5}$ if $a \in M, b \in M \backslash \mathbb{N}$ and $a \times_{M_{k}} b=c_{k}$ for $k=1,2$ then $c_{1} E_{M_{1}}^{3} c_{2}$ and $c_{1} E_{M}^{5} c_{2}$.

[By 1.13(1) we have $c_{1} E_{M}^{5} c_{2}$ and use $(*)_{0}(a)$ to deduce $c_{1} E_{M_{1}}^{3} c_{2}$.]

$(*)_{6}$ if $M_{\ell} \models " a_{1} \times a_{2} \times \ldots \times a_{n}=b_{\ell}$ " for $\ell=1,2$ then $b_{1} E_{M_{1}}^{3} b_{2}$ and $b_{1} E_{M}^{5} b_{2}$. 
[Why? Similarly to $(*)_{5}$, i.e. by $1.13(2)$.]

$(*)_{7} \quad E_{M_{1}}^{4}=E_{M_{2}}^{4}$.

[Why? Let $a, b \in M \backslash \mathbb{N}$ be given. For $\ell=1,2$ and $n \in \mathbb{N}$ let $a_{\ell, n}$ be such that $M_{\ell} \models " a^{n}=a_{\ell, n} "$.

First, assume $a E_{M_{2}}^{4} b$ and without loss of generality $a<b$. So for some $n \in \mathbb{N}$ we have $M_{2} \models$ " $a<b<a^{n}$ " so $M_{2} \models$ " $a<b<a_{2, n}$ ". Also $a_{1, n} E_{M_{1}}^{3} a_{2, n}$ by $(*)_{6}$ so by 2.1(1) we have $a_{1, n} E_{M_{1}}^{4} a_{2, n}$ hence for some $m, M_{1} \models$ " $\left(a_{1, n}\right)^{m} \geq a_{2, n}$ ", in fact, even $m=2$ is O.K. So $M_{1} \models$ " $b<a_{1, m n}$ " but $a<_{M} b$, so together $a E_{M_{1}}^{4} b$.

Second, assume $\neg\left(a E_{M_{2}}^{4} b\right)$ and without loss of generality $a<_{M} b$. So for every $n \in \mathbb{N}, a_{2, n}<_{M} b$ and by $(*)_{6}$ we have $a_{2, n} E_{M_{1}}^{3} a_{1, n}$ hence $a_{1, n} / E_{M_{1}}^{3}$ has a member $<b$, and so in particular $a_{1, n+1} / E_{M_{1}}^{3}$ has a member $<b$, but $a_{1, n} / E_{M_{1}}^{3}$ is below $a_{1, n+1} / E_{M_{1}}^{3}$ (just think on the definitions) so $a_{1, n}<_{M} b$. As this holds for every $n \in \mathbb{N}$ we conclude $\neg\left(a E_{M_{1}}^{4} b\right)$.]

Let

$(*)_{8} I_{d}^{k}=\left\{c \in M: M_{\ell} \models " c^{n}<d\right.$ " for every $\left.n \in \mathbb{N}\right\}$ for $d \in M$ and $k=1,2$.

Now

$(*)_{9} I_{d}^{1}=I_{d}^{2}$ for $d \in M$.

[Why? By $(*)_{7}$ as $I_{d}^{\ell}=\left\{c: c / E_{M_{\ell}}^{4}\right.$ is below $\left.d\right\}$.]

$(*)_{10} \quad E_{M_{2}}^{3}=E_{M_{1}}^{3}$.

[First, if $a_{1} E_{M_{2}}^{3} a_{2}$ then by 1.5)(3), (4) we have $a_{1} E_{M_{2}}^{5} a_{2}$ which, recalling $M_{1} \uparrow\{<\}=$ $M=M_{2} \uparrow\{<\}$, is equivalent to $a_{1} E_{M_{1}}^{5} a_{2}$ which implies (by $M_{1}$ being 3 -o.r. which we are assuming) $a_{1} E_{M_{1}}^{3} a_{2}$.

Second, assume $\neg\left(a_{1} E_{M_{2}}^{3} a_{2}\right)$ and without loss of generality $a_{1}<_{M} a_{2}$. As $M_{2} \models$ PA, for some $c \in M$ we have $M_{2} \models$ "cal $\leq a_{2}<(c+1) a_{2}$ " so by the previous sentence $c \notin I_{a_{1}}^{2}$ hence by $(*)_{9}$ also $c \notin I_{a_{1}}^{1}$.

By $(*)_{5}$ we have $\left(c \times_{M_{1}} a_{1}\right) E_{M_{1}}^{3}\left(c \times_{M_{2}} a_{1}\right)$ and as $c \times_{M_{2}} a_{1} \leq_{M} a_{2}$ clearly $\left(c \times_{M_{2}} a_{1}\right) / E_{M_{1}}^{3} \leq a_{2} / E_{M_{1}}^{3}$ so together $\left(c \times_{M_{1}} a_{1}\right) / E_{M_{1}}^{3}$ is smaller or equal to $a_{2} / E_{M_{1}}^{3}$ so for some $a_{3} \in a_{2} / E_{M_{1}}^{3}$ we have $c \times_{M_{1}} a_{1}<a_{3}$. As $c \notin I_{a_{1}}^{1}$ this implies $a_{1}, a_{3}$ are not $E_{M_{1}}^{3}$-equivalent, so by the choice of $a_{3}$ also $\neg\left(a_{1} E_{M_{1}}^{3} a_{2}\right)$, so we are done proving $(*)_{10}$.]

Hence by $(*)_{7}+(*)_{10}$ part (1) holds.

2),3) By part (1) and 2.7 below for the function $x \mapsto 2^{2^{x}}$, and the equivalence relation $E_{M}^{4}$.

Claim 2.7. The models $M_{1}, M_{2}$ are almost $\{<,+\}$-isomorphic when

(a) $E_{k}$ is a convex equivalence relation on $M_{k} \backslash \mathbb{N}$ for $k=1,2$

(b) $h$ is an order-isomorphism from $M_{1}$ onto $M_{2}$ mapping $E_{1}$ onto $E_{2}$

(c) $f_{k}$ is a function definable in $M_{k}$, is increasing, maps $\mathbb{N}$ into $\mathbb{N}$, for each $E_{k}$-equivalence class $Y$ the set $\left\{a \in M: f_{k}(a) \in Y\right\}$ has the order type of $\mathbb{Z}$ and is unbounded from below and from above in $Y$, for $k=1,2$, of course

(d) for $k=1,2$, if $a_{1} E_{k} a_{2}$ and $b_{1} E_{k} b_{2}$ then $\left(a_{1}+b_{1}\right) E_{k}\left(a_{2}+b_{2}\right)$.

Proof. As in the proof of 1.8 
Claim 2.8. Assume $h$ is an order-isomorphism from $M_{1}$ onto $M_{2}$ and $M_{1}$ is 4-o.r. $\underline{T h e n}$ for $a, b \in M$ we have $h(a) E_{M_{2}}^{4} h(b) \Rightarrow a E_{M_{1}}^{4} b$.

Proof. Without loss of generality $h$ is the identity and let $M_{1} \uparrow\{<\}=M=M_{2} \uparrow$

$\{<\}$. Define $E_{M}=\left\{(a, b)\right.$ : there are $n \in \mathbb{N}, c_{1} \in M, c_{2} \in M$ such that $c_{1} \leq_{M}$ $\min _{M}\{a, b\}$ and $\max _{M}\{a, b\} \leq c_{2}$ and $\left.\left(M_{<c_{1}}\right)^{n} \cong M_{<c_{2}}\right\}$. Easily $E_{M}$ is a convex equivalence relation, $E_{M_{\ell}}^{4} \subseteq E_{M}$ for $\ell=1,2$ and $E_{M_{1}}^{4}=E_{M}$.

Claim 2.9. 1) Assume $a \in M$ is non-standard and $I=\left\{b / E_{M}^{3}: b \in a / E_{M}^{4}\right\}$, naturally ordered. Then the linear order $I$ can be embedded into $\mathbb{R}_{>0}$ with dense image.

2) If $M$ is $\aleph_{1}$-saturated then the embedding is onto $\mathbb{R}_{>0}$.

3) Moreover defining $+_{I}$ by $\left(b_{1} / E_{M}^{3}\right)+_{I}\left(b_{2} / E_{M}^{3}\right)=\left(b_{3} / E_{M}^{3}\right)$ when $b_{1} \times_{M} b_{2}=b_{3}$, the embedding commute with addition so the image is an additive sub-semi-group of $\mathbb{R}$. Also $1_{\mathbb{R}}$ belongs to the image.

Proof. As in Definition 3.2 but we elaborate.

Fix $a \in M$ and for $b \in a / E_{M}^{4}$ let $\mathscr{S}_{a, b}=\left\{\frac{m_{1}}{m_{2}}: m_{1}, m_{2} \in \mathbb{N} \backslash\{0\}\right.$ and $M \models$ " $\left.b^{m_{2}} \geq a^{m_{1}} "\right\}$. Clearly $S_{b}$ is a subset of $\mathbb{Q}_{>0}$ and as $M$ is a model of PA clearly $S_{b}$ is an initial segment of $\mathbb{Q}_{>0}$. By the definition of " $b \in a / E_{M}^{4}$ necessarily $S_{b} \neq \emptyset$ and $S_{b} \neq \mathbb{Q}_{>0}$, so together $r_{b}=\sup \left(\mathscr{S}_{b}\right)$ belongs to $\mathbb{R}_{>0}$.

Again by PA

(a) $r_{b_{1}} \leq r_{b_{2}}$ when $b_{1} \leq_{M} b_{2}$ are from $a / E_{M}^{4}$.

[Why? See the definition of $\mathscr{S}_{b_{1}}, S_{b_{2}}$.]

(b) $r_{b_{1}}=r_{b_{1}} \Leftrightarrow b_{1} E_{M}^{3} b_{2}$ for $b_{1}, b_{2} \in a / E_{M}^{4}$.

[Why? By the definition of $E_{M}^{3}$.]

(c) if $\mathbb{Q} \models$ " $\frac{m_{1}}{m_{2}}<\frac{m_{3}}{m_{4}}$ " where $m_{\ell} \in \mathbb{N} \backslash\{0\}$ for $\ell=1,2,3,4$ then for some $b \in a / E_{M}^{4}$ we have $\mathbb{R} \models$ “ $\frac{m_{1}}{m_{2}} \leq r_{b}<\frac{m_{3}}{m_{4}} "$.

[Why? Let $n \in \mathbb{N} \backslash\{0\}$ be such that $M \models " b<a^{n}$ ", exists as $b \in a / E_{M}^{4}$. Without loss of generality $m_{2}=m_{4}$ cal it $m$, so necessarily $m_{1}<m_{3}$ and without loss of generality $m_{1}+n<m_{3}$. Now by the definition of $b \mapsto r_{b}$ the demand on $b$ means that $M \models " b^{m} \geq a^{m_{1}}$ and $b^{m}<a^{m_{3}}$ ". Let $b$ be the minimal member of $M$ such that $M \models " b^{m} \geq a^{m_{1}}$ " hence $M \models " b^{m-1}<a^{m_{1}}$ hence $b^{m}<a^{m_{1}} b \leq a^{m_{1}} a^{n}=$ $a^{m_{1}+n} \leq a^{m_{3}} "$ so $b$ is as required.]

(d) $\left\{r_{b}: b \in a / E_{M}^{4}\right\}$ is a dense subset of $\mathbb{R}_{>0}$.

[Why? By (c).]

(e) If $M$ is $\aleph_{1}$-saturated then $\left\{r_{b}: b \in a / E_{M}^{4}\right\}=\mathbb{R}_{>0}$, i.e. part (2).

[Why? For any real $r$ and $n$ we can find $b_{1}, b_{2} \in a / E_{M}^{4}$ such that $r-\frac{1}{n}<r_{b_{2}}<$ $r<r_{b_{2}}<\frac{1}{n}$ by $(\mathrm{d})$ and "QQQ is dense in $\mathbb{R}$ ".]

$(f)$ part (3) of the claim holds.

[Why? By the rules of exponentiation which can be phrased in PA.]

Together we are done.

Remark 2.10. So the "distance" between $E_{M}^{3}$ and $E_{M}^{4}$ is small. 


\section{$\S 3$. Constructing somewhat Rigid models}

Hypothesis 3.1. $\lambda$ is regular.

Definition 3.2. For any $M$ (model of PA)

(a) let $\mathbb{Z}_{M}=\mathbb{Z}[M]$ be the ring $M$ generates (so $a \in \mathbb{Z}_{M}$ iff $a=b \vee a=-b$ for some $b \in M$, of course $\mathbb{Z}_{M}$ is determined only up to isomorphism over $M$; similarly below); when, as usual, $M$ is ordinary without loss of generality $\mathbb{Z}_{M} \supseteq \mathbb{Z}$

(b) let $\mathbb{Q}_{M}=\mathbb{Q}[M]$ be the field of quotients of $\mathbb{Z}_{M}$; in fact, it is an ordered field, if $M$ is ordinary then without loss of generality $\mathbb{Q}_{M} \supseteq \mathbb{Q}$

(c) let $\mathbb{R}_{M}=\mathbb{R}[M]$ be the closure of $\mathbb{Q}_{M}$ adding all definable cuts, so in particular it is a real closed field, see 3.3 below

(d) let $S_{M}=\mathbb{R}_{M}^{\mathrm{bd}} / \mathbb{R}_{M}^{\mathrm{infi}}$ where (bd stands for bounded, infi stands for infitesimal)

- $\mathbb{R}_{M}^{\mathrm{bd}}=\left\{a \in \mathbb{R}_{M}: \mathbb{R}_{M} \models-n<a<n\right.$ for some $\left.n \in \mathbb{N}\right\}$

- $\mathbb{R}_{M}^{\text {infi }}=\left\{a \in \mathbb{R}_{M}^{\mathrm{bd}}: \mathbb{R}_{M} \models\right.$ " $-1 / n<a<1 / n$ " for every $\left.n \in \mathbb{N}\right\}$

- $\mathbf{j}_{M}$ is the function from $\mathbb{R}_{M}^{\text {bd }}$ into $\mathbb{R}$ such that $M \models " n_{1} / m_{1}<a<$ $n_{2} / m_{2} " \Rightarrow \mathbb{R}=" n_{1} / m_{1}<\mathbf{j}_{M}(a)<n_{2} / m_{2}$ for $n_{1}, n_{2}, m_{1}, m_{2} \in \mathbb{Z}$ " such that $m_{1}, m_{2}>0$.

Remark 3.3. 1) On 3.2(d) see Claim 2.9.

2) Concerning 3.2 (c) note that $\mathbb{R}[M]$ is a sub-field of the Scott-Cauchy completion $\mathbb{R}[M]$ of $\mathbb{Q}[M]$ and that for so called "rather classless" models $M, \mathbb{R}[M]$ coincide with $\overline{\mathbb{R}}[M]$. See more on completions in [Sh:757].

Definition 3.4. Let $\mathrm{AP}=\mathrm{AP}_{\lambda}$ be the set of a such that

(a) $\mathbf{a}=(M, \Gamma)=\left(M_{\mathbf{a}}, \Gamma_{\mathbf{a}}\right)$ but we may 1 write $M_{<b}^{\mathbf{a}}$ instead $\left(M_{\mathbf{a}}\right)_{<b}$

(b) $M$ is a model of PA

(c) $|M|$, the universe of $M$, is an ordinal $<\lambda^{+}$

(d) $\Gamma$ is a set of $\leq \lambda$ of types over $M$

(e) each $p \in \Gamma$ has the form $\left\{a_{p, \alpha}<x<b_{p, \alpha}: \alpha<\lambda\right\}$ where $\alpha<\beta \Rightarrow M \models$ $a_{p, \alpha}<a_{p, \beta}<b_{p, \beta}<b_{p, \alpha}$

(f) $M$ omits every $p \in \Gamma$.

Definition 3.5. 1) $\leq_{\mathrm{AP}}$ is the following two-place relation on AP:

$\mathbf{a} \leq_{\mathrm{AP}} \mathbf{b}$ iff $M_{\mathbf{a}} \prec M_{\mathbf{b}}$ and $\Gamma_{\mathbf{a}} \subseteq \Gamma_{\mathbf{b}}$.

2) Let $\mathrm{AP}_{T}=\left\{\mathbf{a} \in \mathrm{AP}: M_{\mathbf{a}}\right.$ is a model of $\left.T\right\}$.

3) Let $\mathrm{AP}^{\text {sat }}=\left\{\mathbf{a} \in \mathrm{AP}: M_{\mathbf{a}}\right.$ is saturated $\}$ and $\mathrm{AP}_{T}^{\text {sat }}=\mathrm{AP}_{T} \cap \mathrm{AP}^{\text {sat }}$.

Claim 3.6. 1) $\leq_{\mathrm{AP}}$ is a partial order of AP.

2) If $\left\langle\mathbf{a}_{\alpha}: \alpha<\delta\right\rangle$ is $\leq_{\mathrm{AP}}$-increasing, $\delta$ a limit ordinal $<\lambda^{+} \underline{\text { then }} \mathbf{a}_{\delta}=\cup\left\{\mathbf{a}_{\alpha}\right.$ : $\alpha<\delta\}$ defined by $M_{\mathbf{a}_{\delta}}=\cup\left\{M_{\mathbf{a}_{\alpha}}: \alpha<\delta\right\}, \Gamma_{\mathbf{a}_{\delta}}=\cup\left\{\Gamma_{\mathbf{a}_{\alpha}}: \alpha<\delta\right\}$, is a $\leq_{\mathrm{AP}}-$ lub of $\left\langle\mathbf{a}_{\alpha}: \alpha<\delta\right\rangle$.

3) Assume $\lambda=\lambda^{<\lambda}>\aleph_{0}$. If $\mathbf{a} \in \mathrm{AP}$ then there is $\mathbf{b}$ such that $\mathbf{a} \leq{ }_{\mathrm{AP}} \mathbf{b}$ and $M_{\mathbf{b}}$ is saturated (of cardinality $\lambda$ ).

Proof. Easy.

\footnotetext{
${ }^{1}$ alternatively, replace $M_{b}^{0}$ by $\left(M_{\mathbf{a}}\right)_{<b}$ in the proof of 3.7 below.
} 
Main Claim 3.7. 1) If (A) then $(B)$ where:

(A) (a) $\lambda=\aleph_{0}, \mathbf{a} \in \mathrm{AP}$

(b) $\quad M_{\mathbf{a}} \models " a_{*}>b_{*}>n$ " for $n \in \mathbb{N}$ and $a_{*}, b_{*}$ are not $E_{M_{\mathbf{a}}}^{3}$-equivalent

(c) $F$ is an order isomorphism from $M_{<a_{*}}^{\mathrm{a}}$ onto $M_{<b_{*}}^{\mathrm{a}}$

(B) there are $\mathbf{b}, c_{*}$ satisfying

(a) $\mathbf{a} \leq \mathrm{AP} \mathbf{b}$

(b) $c_{*}<_{M_{\mathbf{b}}} a_{*}$ so $c_{*} \in M_{\mathbf{b}}$ but $c_{*} \notin M_{\mathbf{a}}$

(c) some $p \in \Gamma_{\mathbf{b}}$ is equivalent to $\left\{F\left(a_{1}\right)<x<F\left(a_{2}\right): a_{1}, a_{2} \in M_{\mathbf{a}}\right.$ and $\left.M_{\mathbf{b}} \models a_{1}<c_{*}<a_{2} \leq a_{*}\right\}$ recalling $F$ is the isomorphism from $(A)(c)$.

Remark 3.8. 1) We use $a_{*}, b_{*}, c_{*}$ as they are constant during the proof of 3.7, and we like to let $a, a_{i}$, etc. be free to denote other things.

2) Below in the Discussion 3.9 we try to explain the proof of 3.7 of course, it cannot be fully digested per se, but the reader can try to look at it from time to time, particularly when you lose track in the proof, hopefully this will help.

\section{Proof. Stage A:}

Let

$\boxplus_{1}(a) \quad \Phi=\Phi_{\mathrm{a}}$ is the set of formulas $\varphi(x)=\varphi(x, \bar{a})$ with $\varphi(x, \bar{y}) \in \mathbb{L}\left(\tau_{\mathrm{PA}}\right)$ and $\bar{a} \in{ }^{\ell g(\bar{y})}\left(M_{\mathbf{a}}\right)$

(b) $\varphi^{\prime}(x) \vdash \varphi^{\prime \prime}(x)$ means both are from $\Phi$ and

$M_{\mathbf{a}} \models "(\forall x)\left(\varphi^{\prime}(x) \rightarrow \varphi^{\prime \prime}(x) "\right.$

(c) $\varphi\left(M_{\mathbf{a}}\right)=\varphi\left(M_{\mathbf{a}}, \bar{a}\right)=\left\{b \in M_{\mathbf{a}}: M_{\mathbf{a}} \models \varphi[b, \bar{a}]\right\}$ if $\varphi=\varphi(x)=$ $\varphi(x, \bar{a}) \in \Phi$

(d) we define $\Sigma_{\mathbf{a}}^{k}=\Sigma_{M_{\mathrm{a}}}^{k}$ as the set of $\sigma\left(x_{0}, \ldots, x_{k-1}\right)=\sigma\left(x_{0}, \ldots, x_{k-1}, \bar{a}\right)$ satisfying

$\sigma(\bar{x}, \bar{y})$ is a definable function in $M_{\mathbf{a}}$ and $\bar{a} \in{ }^{\ell g(\bar{y})}\left(M_{\mathbf{a}}\right)$

and $k \in \mathbb{N}$, we may omit $k$ when it is 1 and so may write $\sigma(x, \bar{y})$ and $\sigma(x)$

(e) $\quad \operatorname{let2} \xi(\varphi)=\xi(\varphi(x))=\mathbf{j}_{M}\left(\log _{2}\left(\left|\varphi\left(M_{\mathbf{a}}\right)\right|\right) / \log \left(a_{*}\right)\right)$ for $\varphi=\varphi(x) \in \Phi_{\mathbf{a}}$ such that $\varphi(M) \subseteq\left[0, a_{*}\right)_{M}$, see $3.2(\mathrm{~d})$

(f) if $\varphi_{1}, \varphi_{2} \in \Phi, \varphi_{2}(M) \neq \emptyset$ and $\sigma \in \Sigma_{\mathbf{a}}$ then $^{3}$ let $\sigma^{\prime}:=\sigma\left[\varphi_{1}, \varphi_{2}\right]$ be the following function from $\varphi_{1}(M)$ to $\varphi_{2}(M) \cup\{0\}$, definable in $M: M \models \sigma^{\prime}(a)=b$ iff $a \in \varphi_{1}(M)$ and $b=\max \{b$ : either $b=0$ and $\sigma(a)<\min \left(\varphi_{2}(M)\right)$ or $b \in \varphi_{2}(M)$ and $\left.b \leq \sigma(a)\right\}$.

We define $\mathbb{P}$, it will serve as a set of approximations to $\operatorname{tp}\left(c_{*}, M_{\mathbf{a}}, M_{\mathbf{b}}\right)$, as follows:

$\boxplus_{2}$ the quasi-order $\mathbb{P}$ is defined by:

(a) a member of $\mathbb{P}$ is a pair $\bar{\varphi}=\left(\varphi_{1}, \varphi_{2}\right)$ such that:

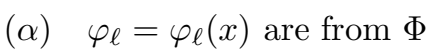

( $\beta) \quad \varphi_{1}(x) \vdash x<a_{*}$ and $\varphi_{2}(x) \vdash x<b_{*}$

$(\gamma) \quad$ if $a_{1}<a_{2}$ are from $\varphi_{1}\left(M_{\mathbf{a}}\right)$ then $\left[F\left(a_{1}\right), F\left(a_{2}\right)\right]_{M} \cap \varphi_{2}(M) \neq \emptyset$

\footnotetext{
2 the $\left|\varphi\left(M_{\mathbf{a}}\right)\right|$ and $\log _{2}$ has natural meanings; of course we can translate it to a formula in $\mathbb{L}\left(\tau_{\mathrm{PA}}\right)$.

${ }^{3} \mathrm{So} \sigma^{\prime}$ is $\sigma$ restricting the domain to $\varphi_{1}(M)$ and rounding the image to be in $\varphi_{2}(M) \cup\{0\}$.
} 


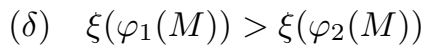

(b) $\mathbb{P} \models \bar{\varphi}^{\prime} \leq \bar{\varphi}^{\prime \prime}$ iff $\varphi_{\ell}^{\prime \prime}(x) \vdash \varphi_{\ell}^{\prime}(x)$, for $\ell=1,2$.

Note that $\left(\varphi_{1}(x, \bar{a}), \varphi_{2}\left(x, \bar{a}_{2}\right)\right) \in \mathbb{P}$ is not definable in $M_{\mathbf{a}}$ mainly because of $(a)(\gamma)$ of $\boxplus_{2}$.

Obviously observe

$\boxplus_{3}$ if $\bar{\varphi} \in \mathbb{P}$ and $\varphi(x) \in \Phi$ then for some $\mathbf{t} \in\{0,1\}$ we have $\left(\varphi_{1}(x) \wedge\right.$ $\left.\varphi(x)^{\mathbf{t}}, \varphi_{2}(x)\right) \in \mathbb{P}$ (and is $\leq_{\mathbb{P}^{-a b o v e}} \bar{\varphi}$; recall that $\varphi^{\mathbf{t}}$ is $\varphi$ is $\mathbf{t}=1$ and is $\neg \varphi$ if $\mathbf{t}=0)$.

[Why? $M_{\mathbf{a}} \models$ " $\left|\varphi_{1}(M) \cap \varphi(M)\right| \geq \frac{1}{2}\left|\varphi_{1}(M)\right|$ or $M_{\mathbf{a}} \models "\left|\varphi_{1}(M) \backslash \varphi(M)\right| \geq \frac{1}{2}\left|\varphi_{2}(M)\right|$ ".

As $a_{*} \notin \mathbb{N}$ clearly $\xi\left(\varphi_{1}(x) \wedge \varphi(x)\right)=\xi\left(\varphi_{2}(x)\right)$ or $\xi\left(\varphi_{2}(x) \wedge \neg \varphi(x)\right)=\xi\left(\varphi_{2}(x)\right)$. So clearly we are done proving $\boxplus_{3}$.]

$\underline{\text { Stage B: }}$

We arrive at a major point: how to continue to omit members of $\Gamma_{\mathbf{a}}$

$\boxplus_{4}$ if $\bar{\varphi} \in \mathbb{P}, \sigma(x) \in \Sigma_{\mathbf{a}}$ and $p(x) \in \Gamma_{\mathbf{a}}$ then for some $\bar{\varphi}^{\prime}$ and $n$ we have

(a) $\bar{\varphi} \leq \bar{\varphi}^{\prime} \in \mathbb{P}$

(b) $\varphi_{0}^{\prime}(x) \vdash " \sigma(x) \notin\left(a_{p, n}, b_{p, n}\right) "$.

The rest of this stage is dedicated to proving this. We use a "wedge question".

Case 1: There is $d \in M_{\mathbf{a}}$ such that $\bar{\varphi}^{\prime}:=\left(\varphi_{1}(x) \wedge \sigma(x)=d, \varphi_{2}(x)\right) \in \mathbb{P}$.

In this case obviously $\bar{\varphi} \leq \bar{\varphi}^{\prime} \in \mathbb{P}$. Also as $d \in M_{\mathbf{a}}$ and $M_{\mathbf{a}}$ omit $p(x)$ recalling $p(x) \in \Gamma_{\mathbf{a}}$, clearly $d$ does not realize $p(x)$ hence for some $n, d \notin\left(a_{p, n}, b_{p, n}\right)_{M_{\mathbf{a}}}$; so $\bar{\varphi}^{\prime}, n$ are as promised.

$\underline{\text { Case 2: }}$ Not case 1 .

So

$(*)_{4.1} \xi(\varphi(x) \wedge \sigma(x)=d) \leq \xi\left(\varphi_{2}(x)\right)$ for every $d$ from $M_{\mathbf{a}}$.

Clearly there is a minimal $d_{*} \in M_{\mathbf{a}}$ satisfying

$$
(*)_{4.1} \quad M_{\mathbf{a}} \models "\left|\left\{c \in \varphi_{1}(M): \sigma(c) \leq d_{*}\right\}\right| \geq \frac{1}{2}\left|\varphi_{1}\left(M_{\mathbf{a}}\right)\right| " .
$$

So

$$
(*)_{4.2} \quad M_{\mathbf{a}} \models "\left|\left\{c \in \varphi_{1}(M): \sigma(c) \geq d_{*}\right\}\right| \geq \frac{1}{2}\left|\varphi_{2}\left(M_{\mathbf{a}}\right)\right| " .
$$

But $M_{\mathbf{a}}$ omits the type $p(x)$ as $p(x) \in \Gamma_{\mathbf{a}}$ and $d_{*} \in M_{\mathbf{a}}$, so for some $n, d_{*} \notin$ $\left(a_{p, n}, b_{p, n}\right)$.

So one of the following sub-cases occurs:

Sub-case 2a: $d_{*} \leq a_{p, n}$.

Let $\varphi_{1}^{\prime}(x)=\varphi_{1}(x) \wedge\left(\sigma(x) \leq a_{p, n+1}\right)$ and $\varphi_{2}^{\prime}(x)=\varphi_{2}(x)$. Now the pair $\bar{\varphi}^{\prime}=$ $\left(\varphi_{1}^{\prime}, \varphi_{2}^{\prime}\right)$ is as required, noting (by $\left.(*)_{4.1}\right)$ that

$$
M_{\mathbf{a}} \models "\left|\varphi_{1}^{\prime}(M)\right| \geq\left|\left\{a \in \varphi_{1}(M): \sigma(c) \leq d_{*}\right\}\right| \geq \frac{1}{2}\left|\varphi_{1}(M)\right| "
$$

hence

$$
\xi\left(\varphi_{1}^{\prime}(x)\right)=\xi\left(\varphi_{1}(x)\right)>\xi\left(\varphi_{2}(x)\right)=\xi\left(\varphi_{2}^{\prime}(x)\right) .
$$


Sub-case $2 \mathrm{~b}: d_{*} \geq a_{p, n}$.

Let $\varphi_{1}^{\prime}(x)=\varphi_{2}(x) \wedge\left(\sigma(x) \geq b_{p, n+1}\right)$ and $\varphi_{2}^{\prime}(x)=\varphi_{2}(x)$. Now $\bar{\varphi}^{\prime}=\left(\varphi_{1}^{\prime}, \varphi_{2}^{\prime}\right)$ is as required noting (by $(*)_{4.2}$ ) that

$$
M_{a} \models "\left|\varphi_{1}^{\prime}(M)\right| \geq\left|\left\{c \in \varphi_{1}(M): \sigma(c) \geq d_{*}\right\}\right| \geq \frac{1}{2}\left|\varphi_{1}(M)\right| "
$$

hence

$$
\xi\left(\varphi_{1}^{\prime}(x)\right)=\xi\left(\varphi_{1}(x)\right)>\xi\left(\varphi_{2}(x)\right)=\xi\left(\varphi_{2}^{\prime}(x)\right) .
$$

So we are done proving $\boxplus_{4}$.

\section{Stage C:}

How do we omit the new type? Recall that $c_{*}$ will realize a type to which $\bar{\varphi} \in \mathbb{P}$ is an approximation and we have to omit the relevant type from clause $(B)(c)$ of the Claim.

This stage is dedicated to proving the relevant statement:

$\boxplus_{5}$ if $\bar{\varphi} \in \mathbb{P}$ and $\sigma(x) \in \Sigma_{\mathbf{a}}$ then for some $\bar{\varphi}^{\prime}$ :

(a) $\bar{\varphi} \leq \bar{\varphi}^{\prime} \in \mathbb{P}$

(b) for some $a_{1}<a_{2} \leq a_{*}$ we have

$$
\begin{array}{ll}
\text { - } & \varphi_{1}^{\prime}(x) \vdash a_{1} \leq x<a_{2} \\
\text { - } & \varphi_{1}^{\prime}(x) \vdash \neg\left(F\left(a_{1}\right) \leq \sigma(x)<F\left(a_{2}\right)\right) .
\end{array}
$$

First note

$(*)_{5.1}$ if there is $\bar{\varphi}^{\prime} \in \Phi$ such that $\bar{\varphi} \leq \bar{\varphi}^{\prime}$ and $\xi\left(\bar{\varphi}_{1}^{\prime}\right) / \xi\left(\bar{\varphi}_{2}^{\prime}\right)>2$ then the conclusion of $\boxplus_{5}$ holds.

[Why? As $\left|\bigcup_{i} A_{i}\right|=\sum_{i}\left|A_{i}\right|$ for pairwise disjoint sets, i.e. the version provable in PA we can find $\varphi_{1}^{\prime \prime}(x) \in \Phi$ such that $\varphi_{1}^{\prime \prime}\left(M_{\mathbf{a}}\right) \subseteq \varphi_{1}^{\prime}\left(M_{\mathbf{a}}\right), M_{\mathbf{a}} \models "\left|\varphi_{1}^{\prime}\left(M_{\mathbf{a}}\right)\right| /\left|\varphi_{1}^{\prime \prime}\left(M_{\mathbf{a}}\right)\right| \leq$ $\left|\varphi_{2}\left(M_{\mathbf{a}}\right)\right| "$ and $\sigma\left[\varphi_{1}^{\prime \prime}, \varphi_{2}\right]$, which was defined in Clause $\boxplus_{1}(f)$, is constant say constantly $e$, hence $e \in \varphi_{2}\left(M_{\mathbf{a}}\right)$. So $\xi\left(\varphi_{1}^{\prime \prime}(x)\right) \geq \xi\left(\varphi_{1}^{\prime}(x)\right)-\xi\left(\varphi_{2}^{\prime}(x)\right)>2 \xi\left(\varphi_{2}^{\prime}(x)\right)-$ $\xi\left(\varphi_{2}^{\prime}(x)\right)=\xi\left(\varphi_{2}^{\prime}(x)\right)$ hence $\left(\varphi_{1}^{\prime \prime}, \varphi_{2}^{\prime}\right)$ belongs to $\mathbb{P}$ and $\bar{\varphi} \leq \bar{\varphi}^{\prime} \leq\left(\varphi_{1}^{\prime \prime}, \varphi_{2}^{\prime}\right)$. As $e \in \varphi_{2}\left(M_{\mathbf{a}}\right) \subseteq\left[0, b_{*}\right)_{M_{\mathbf{a}}}$ and $F$ is onto $M_{<b_{*}}^{\mathbf{a}}$ for some $d<_{M_{\mathbf{a}}} a_{*}$ we have $F(d)=e$. By $\boxplus_{3}$ without loss of generality $\varphi^{\prime \prime}(x) \vdash$ " $x<d$ " or $\varphi^{\prime \prime}(x) \vdash$ " $d \leq x$ " so we can choose $\left(a_{1}, a_{2}\right)$ as $(0, d)$ or as $\left(d, a_{*}\right)$. So we are done.]

So we can assume $(*)_{5.1}$ does not apply. Hence it is natural to deduce (can replace $\frac{1}{8}$ by any fixed $\varepsilon>0$ ).

$(*)_{5.2}$ Without loss of generality for no $\bar{\varphi}^{\prime} \in \Phi$ do we have $\bar{\varphi} \leq \bar{\varphi}^{\prime}$ and

$$
\xi\left(\varphi_{1}^{\prime}\right) / \xi\left(\varphi_{2}^{\prime}\right)>\left(1+\frac{1}{8}\right) \xi\left(\varphi_{1}\right) / \xi\left(\varphi_{2}\right) .
$$

[Why? We try to choose $\bar{\varphi}^{n}$ by induction on $n \in \mathbb{N}$ such that $\bar{\varphi}^{n} \in \mathbb{P}, \bar{\varphi}^{0}=$ $\bar{\varphi}, \bar{\varphi}^{n} \leq \bar{\varphi}^{n+1}$ and $\xi\left(\varphi_{1}^{n}\right) / \xi\left(\varphi_{2}^{\prime}\right) \geq\left(1+\frac{1}{8}\right)^{n} \xi\left(\varphi_{1}\right) / \xi\left(\varphi_{2}\right)$. So for some $n$ we have $\xi\left(\varphi_{1}^{n}\right) / \xi\left(\varphi_{2}^{\prime}\right)>2$ and we can apply $(*)_{5.1}$, contradiction. But $\bar{\varphi}^{0}$ is well defined, hence for some $n, \bar{\varphi}^{n}$ is well defined but we cannot choose $\bar{\varphi}^{n+1}$. Now $\bar{\varphi}^{n}$ is as required in $(*)_{5.2}$.]

Clearly 
$(*)_{5.3}$ if $a_{1}<a_{2}$ are from $\varphi_{1}(M)$, so $b_{1} \leq F\left(a_{1}\right)<F\left(a_{2}\right) \leq b_{2}$ are from $\varphi_{2}(M)$ and $\xi\left(\varphi_{1}(x) \wedge a_{1} \leq x<a_{2} \wedge \sigma(x) \notin\left[b_{1}, b_{2}\right)\right)>\xi\left(\varphi_{2}(x) \wedge b_{1} \leq x<b_{2}\right)$ then we are done.

So from now on we assume that there are no $a_{1}, a_{2}$ as in $(*)_{5.3}$.

Let

$(*)_{5.4}(a) \quad k_{*} \in \mathbb{N} \backslash\{0\}$ be large enough such that $\left(\xi\left(\varphi_{1}\right)-\xi\left(\varphi_{2}\right)\right) / \xi\left(\varphi_{2}\right)>2 / k_{*}$

(b) let $n(1) \in \mathbb{N}$ be large enough such that:

- $\xi\left(\varphi_{1}\right)-\xi\left(\varphi_{2}\right)>1 / n(1)$

- $\xi\left(\varphi_{2}\right)>\left(k_{*}+1\right) / n(1)$

(c) $\quad$ let $n(2) \in \mathbb{N}$ be $>n(1)$

$(*)_{5.5}$ let

(a) $\quad \psi_{1}\left(x_{1}, x_{2} ; y_{1}, y_{2}\right)=x_{1}<x_{2} \wedge \varphi_{1}\left(x_{1}\right) \wedge \varphi_{1}\left(x_{2}\right) \wedge y_{1}<y_{2}<b_{*}$

(b) $\psi_{2}\left(x_{1}, x_{2} ; y_{1}, y_{2}\right)$ is the conjunction of:

- $\psi_{1}\left(x_{1}, x_{2}, y_{1}, y_{2}\right)$

- $\left|\left\{x: \varphi_{1}(x) \wedge x_{1} \leq x<x_{2}\right\}\right|^{n(1)} \geq\left|\left\{y: \varphi_{2}(y) \wedge y_{1} \leq y<y_{2}\right\}\right|^{n(1)} \times a_{*}$

(c) $\vartheta_{2}\left(x_{1}, x_{2}\right)=\left(\exists y_{1}, y_{2}\right)\left(\psi_{2}\left(x_{1}, x_{2} ; y_{1}, y_{2}\right)\right.$

(d) $\psi_{3}\left(x_{1}, x_{2} ; y_{1}, y_{2}\right)$ is the conjunction of

- $\psi_{2}\left(x_{1}, x_{2} ; y_{1}, y_{2}\right)$

- $\left|\left\{x: \varphi_{1}(x) \wedge x_{1} \leq x<x_{2} \wedge \sigma(x) \notin\left[y_{1}, y_{2}\right)\right\}\right|^{n(2)}<\mid\left\{y: \varphi_{2}(a) \wedge y_{1}\right.$ $\left.\leq y<y_{2}\right\}\left.\right|^{n(2)} \times a_{*}$

(e) $\vartheta_{3}\left(x_{1}, x_{2}\right)=\left(\exists y_{1}, y_{2}\right) \psi_{3}\left(x_{1}, x_{2}, y_{1}, y_{2}\right)$.

So by our assumptions (for clause (b) use " $(*)_{5.3}$ does not apply") we have

$(*)_{5.6}(a) \quad$ if $a_{1}<a_{2}$ are from $\varphi_{1}\left(M_{\mathbf{a}}\right)$ then $M_{\mathbf{a}} \models \psi_{1}\left[a_{1}, a_{2} ; F^{\left[\varphi_{2}\right]}\left(a_{1}\right), F^{\left[\varphi_{2}\right]}\left(a_{2}\right)\right]$

(b) $\quad$ if $M_{\mathbf{a}} \models \psi_{2}\left[a_{1}, a_{2} ; F^{\left[\varphi_{2}\right]}\left(a_{1}\right), F^{\left[\varphi_{2}\right]}\left(a_{2}\right)\right]$ then

- $M_{\mathbf{a}} \models \psi_{3}\left[a_{1}, a_{2} ; F^{\left[\varphi_{2}\right]}\left(a_{1}\right), F^{\left[\varphi_{2}\right]}\left(a_{2}\right)\right]$

- $M_{\mathbf{a}} \models \vartheta_{3}\left[a_{1}, a_{2}\right]$.

Clearly

$(*)_{5.7}$ if $M_{\mathbf{a}} \models \psi_{3}\left[a_{1}, a_{2} ; b_{1}^{\iota}, b_{2}^{\iota}\right]$ for $\iota=1,2$ then $\left[b_{1}^{1}, b_{2}^{1}\right)_{M_{\mathbf{a}}} \cap\left[b_{1}^{2}, b_{2}^{2}\right)_{M_{\mathbf{a}}} \neq \emptyset$.

It is well known that for a linear order, for any finite family of intervals, their intersection is non-empty iff the intersection of any two is non-empty.

Now a version of this can be proved in PA hence

$(*)_{5.8}$ for some $\sigma\left(x_{1}, x_{2}\right) \in \Sigma_{\mathbf{a}}^{2}$, we have: if $M_{\mathbf{a}} \models \vartheta_{3}\left[a_{1}, a_{2}\right] \underline{\text { then }} \sigma\left(a_{1}, a_{2}\right) \in$ $\varphi_{2}(M)$ and for every $b_{1}, b_{2} \in \varphi_{2}(M)$ we have $M_{\mathbf{a}} \models \psi_{3}\left[a_{1}, a_{2} ; b_{1}, b_{2}\right]$ implies $\sigma\left(a_{1}, a_{2}\right) \in\left[b_{1}, b_{2}\right)_{M_{\mathrm{a}}}$.

Now

$(*)_{5.9}$ (a) let $\varepsilon \in \mathbb{Q}_{M} \subseteq \mathbb{R}_{M}$ be a true rational such that

$$
\xi\left(\varphi_{2}\right)>\varepsilon>\xi\left(\varphi_{2}\right) k_{*} /\left(k_{*}+1\right)+1 / n(1)
$$

(b) let $d_{*}=\left\lfloor\left(a_{*}\right)^{\varepsilon}\right\rfloor \in M_{\mathbf{a}}$ computed in $\mathbb{R}_{\mathbf{a}}$ and $c_{*}=\left\lfloor\left(a_{*}\right)^{\varepsilon-1 / n(1)}\right\rfloor$

$(*)_{5.10}$ in $M_{\mathbf{a}}$ we can define an increasing sequence $\left\langle a_{1, i}: i<i(*)\right\rangle$, so $i(*) \in M_{\mathbf{a}}$ such that 
- $a_{1,0}=0, a_{1, i(*)}=a_{*}$

- $a_{1, i+1}=\min \left\{a: \varphi_{1}(a)\right.$ and $a_{1, i}<a$ and $\left|\varphi_{1}\left(M_{\mathbf{a}}\right) \cap\left[a_{1, i}, a\right)\right|$ is $\left.d_{*}\right\}$

$(*)_{5.11}(a) \quad$ in $M_{\mathbf{a}}$ we can define

- $\mid\left\{a: \varphi_{1}(a)\right.$ and $\left.a_{1, i(*)-1} \leq a<a_{*}\right\} \mid$ is $\geq d_{*}$ but $\leq 2 d_{*}$

$$
\begin{aligned}
& u=\left\{i<i(*): M_{\mathbf{a}} \models \vartheta_{3}\left[a_{1, i}, a_{1, i+1}\right]\right\} \\
& v=\{i<i(*): i \notin u\}
\end{aligned}
$$

(b) let $\varphi_{1, i}(x):=\varphi_{1}(x) \wedge a_{1, i} \leq x<a_{1, i+1}$.

So (will be used in Case 1 below)

$(*)_{5.12}(a) \quad \xi\left(\varphi_{1, i}(x)\right)=\varepsilon$ for $i<i(*)$

(b) if $i<i(*)$ and $i \in v$ then

$$
\begin{aligned}
& M_{\mathbf{a}} \models "\left|\varphi_{2}\left(M_{\mathbf{a}}\right) \cap\left(F\left(a_{1, i}\right), F\left(a_{1, i+1}\right)\right)_{M_{\mathbf{a}}}\right| \geq\left|\varphi_{1, i}\left(M_{\mathbf{a}}\right)\right| \times a_{*}^{-1 / n(1)}, \\
& =b_{*} \times a_{*}^{-1 / n(1)}, "
\end{aligned}
$$

[Why? Clause (a) is obvious by the definition of $\xi(-)$ and $a_{1, i+1}$. For clause (b) note that by the definition of $\vartheta_{3}$ in $(*)_{5.5}(e)$ we have $M_{\mathbf{a}} \models \neg \psi_{3}\left[a_{1, i}, a_{2, i} ; F^{\left[\varphi_{2}\right]}\left(a_{1, i}\right), F^{\left[\varphi_{2}\right]}\left(a_{2, i}\right)\right]$, but by $(*)_{5.6}(a)$ we have $M_{\mathbf{a}} \models \psi_{3}\left[a_{1, i}, a_{2, i} ; F\left(a_{1, i}\right), F\left(a_{1, i}\right)\right]$. By the definition of $\psi_{3}$ in $(*)_{5.5}(d)$ we are done.]

Now towards Case 2 note

$(*)_{5.13}$ if $i_{1}<i_{2}$ are from $u$ then $F\left(a_{1, i_{1}}\right)<\sigma^{M}\left(a_{1, i_{2}}, a_{1, i_{2}+1}\right)$.

[Why? Obvious by $(*)_{5.8}$.]

$(*)_{5.14}$ we define terms $\sigma_{1}\left(x_{1}, x_{2}\right), \sigma_{2}\left(x_{1}, x_{2}\right) \in \Sigma_{\mathbf{a}}^{2}$ such that if $i<i(*)$ then:

(a) $M_{\mathbf{a}} \models \sigma_{1}\left(a_{1, i}, a_{1, i+1}\right)<\sigma\left(a_{1, i}, a_{1, i+1}\right)<\sigma_{2}\left(a_{1, i}, a_{1, i+1}\right)$

(b) $\varphi_{2}\left(M_{\mathbf{a}}\right) \cap\left[\sigma^{M_{\mathbf{a}}}\left(a_{1, i}, a_{1, i+1}\right), \sigma_{2}^{M_{\mathbf{a}}}\left(a_{1, i}, a_{1, i+1}\right)\right)_{M_{\mathbf{a}}}$ has $\leq c_{*}$ elements, in $M_{\mathrm{a}}$ 's-sense

(c) $\varphi_{2}\left(M_{\mathbf{a}}\right) \cap\left[\sigma_{1}^{M_{\mathbf{a}}}\left(a_{1, i}, a_{1, i+1}\right), \sigma^{M_{\mathbf{a}}}\left(\left(a_{1, i}, a_{1, i+1}\right)\right)_{M_{\mathbf{a}}}\right.$ has $\leq c_{*}$ elements in $M_{\mathbf{a}}$ 's-sense

(d) if $i<j$ are from $u$ then $M_{\mathbf{a}} \models$ " $\sigma_{2}\left(a_{1, i}, a_{1, i+1}\right)<\sigma\left(a_{1, j}, a_{1, j+1}\right)$ "

(e) $M_{\mathbf{a}} \models$ " $\sigma_{1}\left(a_{1, i}, a_{1, i+1}\right)<\sigma\left(a_{1, i}, a_{1, i+1}\right)<\sigma_{2}\left(a_{1, i}, a_{1, i+1}\right)$ "

$(f)$ if $i \in u$ then $M_{\mathbf{a}} \models$ " $\sigma_{1}\left(a_{1, i}, a_{1, i+1}\right)<F\left(a_{1, i}\right)<\sigma\left(a_{1, i}, a_{1, i+1}\right)<$ $F\left(a_{1, i+1}\right)<\sigma_{2}\left(a_{1, i}, a_{1, i+1}\right) "$.

[Why? Let $\sigma_{2}\left(a_{1, i}, a_{1, i+1}\right)$ be maximal such that the relevant part of (a) and (b),(d) holds and $\sigma_{1}\left(a_{1, i}, a_{1, i+1}\right)$ be minimal such that the other part of (a) and $(c),(e)$ holds. By $(*)_{5.8}$ we can finish.]

Now the proof splits.

Case 1: $M_{\mathbf{a}} \models$ " $|v| \geq i(*) / 3$ ". Here we shall use clause $(\gamma)$ of $\boxplus_{2}(a)$.

Let $v_{1}=\left\{i \in v: M_{\mathbf{a}} \models\right.$ " $|\{j \in v: j<i\}|$ is even $\}$, so $M_{\mathbf{a}} \models$ "| $v_{1} \mid \geq i(*) / 6$ ". Let $\varphi_{1}^{\prime}(x):=\varphi_{1}(x) \wedge(\exists z)\left[z \in v_{1} \wedge x \in\left[a_{1, z}, a_{1, z+1}\right) \wedge \neg(\exists y)\left(\varphi_{2}(y) \wedge y \in\left[a_{1, z}, a_{1, z+1}\right) \wedge y<\right.\right.$ $x)]$.

Let $\varphi_{2}^{\prime}(x):=\varphi_{2}(x) \wedge\left(\right.$ the number $\left|\left\{y: \varphi_{2}(a) \wedge y<x\right\}\right|$ is divisible by $\left.c_{*}\right)$.

Now

$(*)_{5.14}(a) \quad \bar{\varphi}^{\prime}:=\left(\varphi_{1}^{\prime}(x), \varphi_{2}^{\prime}(x)\right) \in \mathbb{P}$

(b) $\xi\left(\varphi_{1}^{\prime}(x)\right)=\xi\left(\varphi_{1}(x)\right)-\varepsilon$ 
(c) $\xi\left(\varphi_{2}^{\prime}(x)\right)=\xi\left(\varphi_{2}(x)\right)-\varepsilon+1 / n(1)$.

So

$$
\begin{aligned}
\xi\left(\varphi_{1}^{\prime}(x)\right) / \xi\left(\varphi_{2}^{\prime}(x)\right) & =\left(\xi\left(\varphi_{1}(x)\right)-\varepsilon\right) /\left(\xi\left(\varphi_{2}(x)-\varepsilon+1 / n(1)\right)\right. \\
& \geq\left(\xi \left(\varphi_{1}(x)-\xi\left(\varphi_{2}(x)\right) /\left(\xi\left(\varphi_{2}\right)-\xi\left(\varphi_{2}\right) k_{*} /\left(k_{*}+1\right)\right)\right.\right. \\
& =\left(k_{*}+1\right)\left(\xi\left(\varphi_{1}(x)\right)-\xi\left(\varphi_{2}(x)\right)\right) / \xi\left(\varphi_{2}\right)>2
\end{aligned}
$$

and we fall under $(*)_{5.1}$ finishing the proof of $\boxplus_{5}$.

$\underline{\text { Case 2: }} M_{\mathbf{a}} \models "|u| \geq i(*) / 3$.

Define in $M_{\mathrm{a}}$

$u_{2}=\left\{i \in u:\right.$ " $\left|\left\{j \in \varphi_{1}(M): j<i\right\}\right|$ is even" $\}$.

So $M_{\mathbf{a}} \models "\left|u_{1}\right| \geq i(*) / 6$ ". Now $M_{\mathbf{a}}$ satisfies

$$
\begin{aligned}
\left|\varphi_{1}(M)\right| \leq(i(*)+1) d_{*} & \leq 7\left|\cup\left\{\varphi_{1}\left(M_{\mathbf{a}}\right) \cap\left[a_{1, i}, a_{1, i+1}\right): i \in u_{1}\right\}\right| \\
& =7 \Sigma\left\{\left|\varphi_{1}\left(M_{\mathbf{a}}\right) \cap\left[a_{1, i}, a_{1, i+1}\right)\right|: i \in u_{1}\right\} \mid \\
& \leq 7 \Sigma\left\{\left|\varphi_{2}\left(M_{\mathbf{a}}\right) \cap\left[\sigma_{1}\left(a_{1, i}, a_{1, i+1}\right), \sigma_{2}\left(a_{1, i}, a_{1, i+1}\right)\right)\right| \times a_{*}^{1 / n(1)}: i \in u_{1}\right\} \mid \\
& \left.7 \mid \cup\left\{\varphi_{2}, M_{\mathbf{a}}\right) \cap\left[\sigma_{1}\left(a_{1, i}, a_{2, i+1}\right), \sigma_{2}\left(a_{1, i}, a_{1, i+1}\right)\right): i \in u_{2}\right\} \mid \times a_{*}^{1 / n(1)} \\
& <7 \times\left|\varphi_{2}\left(M_{\mathbf{a}}\right)\right| \times a_{*}^{1 / n(1)} .
\end{aligned}
$$

Together $\left|\varphi_{2}(M)\right| \leq 7 \times\left|\varphi_{2}(M)\right| \times a_{a_{*}}^{1 / n(1)}$. But as $n(1)$ was chosen large enough, i.e. $1 / n(1)<\xi\left(\varphi_{2}\left(M_{\mathbf{a}}\right)-\xi\left(\varphi_{2}\left(M_{\mathbf{a}}\right)\right)\right.$, contradiction. So we are done proving $\boxplus_{5}$.

Stage D:

We define the sets $\mathbf{T}=\mathbf{T}_{1} \cup \mathbf{T}_{2} \cup \mathbf{T}_{3}$ of tasks where

$\boxplus_{6} \bullet \mathbf{T}_{1}=\{(1, \varphi(x)): \varphi(x) \in \Phi\}$, toward completeness

- $\mathbf{T}_{2}=\left\{(2, \sigma(x), \varphi(x)): \sigma(x) \in \Sigma_{\mathbf{a}}\right.$ and $\left.p(x) \in \Gamma_{\mathbf{a}}\right\}$, toward preserving $p(x)$ is omitted

- $\mathbf{T}_{3}=\left\{(3, \sigma(x)): \sigma(x) \in \Sigma_{\mathbf{a}}\right\}$, toward "stopping $F$ ", "omitting the new type".

Clearly $\mathbf{T}$ is countable, let $\left\langle\mathbf{s}_{n}: n<\omega\right\rangle$ list it.

We now choose $\bar{\varphi}^{n}$ by induction on $n$ such that:

$\boxplus_{7}(a) \quad \bar{\varphi}^{n} \in \mathbb{P}$

(b) $\bar{\varphi}^{m} \leq \bar{\varphi}^{n}$ for $m<n$

(c) $\quad$ if $n=m+1$ and $\mathbf{s}_{m}=(1, \varphi(x))$ then $\varphi_{n}(x) \vdash \varphi(x)$ or $\varphi_{n}(x) \vdash \neg \varphi(x)$

(d) if $n=m+1$ and $\mathbf{s}_{m}=(2, \sigma(x), p(x))$ then for some $k, \varphi_{n}(x) \vdash$ " $\sigma(x)$ $\notin\left(a_{p, k}, b_{p, k}\right) "$

(e) if $n=m+1$ and $\mathbf{s}_{m}=(3, \sigma(x))$ then for some $a_{1, m}<a_{2, m} \leq a_{*}$ we have $\varphi_{n}^{\prime}(x) \vdash a_{1, m} \leq x<a_{2, m} \wedge \neg\left(F\left(a_{1, n}\right) \leq \sigma(x)<a_{2, m}\right)$.

Why can we carry the induction? $\bar{\varphi}^{0}$ trivial, for $\bar{\varphi}^{m+1}$ if $\mathbf{s}_{m} \in \mathbf{T}_{1}$ by $\boxplus_{3}$, if $\mathbf{s}_{m} \in \mathbf{T}_{2}$ by $\boxplus_{4}$ and if $\mathbf{s}_{m} \in \mathbf{T}_{3}$ by $\boxplus_{5}$, more fully, let $\mathbf{s}_{m}=(3, \sigma(x))$, let $\sigma^{\prime}(x)$ be defined by $\sigma^{\prime}(x)=\min \left\{y: y=b_{*}\right.$ or $\left.\sigma(x) \leq y \wedge \varphi(y)\right\}$, now apply $\boxplus_{5}$ to $\left(\bar{\varphi}^{m}, \sigma^{\prime}(-)\right)$.

Note

$(*)_{7.1}\left\{\varphi_{n}(x): n<\omega\right\}$ is a complete type over $M_{\mathbf{a}}$. 
[Why? By clause (c) of $\boxplus_{7}$ and the choice of $\mathbf{T}_{1}$.]

By compactness there are $N$ and $c_{*}$ such that

$(*)_{7.2}(a) \quad M_{\mathbf{a}} \prec N$

(b) $c_{*}$ realizes $\left\{\varphi_{n}(x): n<\omega\right\}$.

As $T$ being a completion of PA has definable Skolem functions, without loss of generality

$(*)_{7.3} N$ is the Skolem hull of $M_{\mathbf{a}} \cup\left\{c_{*}\right\}$.

Now by $\boxplus_{7}(d)$

$(*)_{7.4} N$ omit every $p \in \Gamma_{\mathbf{a}}$.

Also by $\boxplus_{7}(c)$

$(*)_{7.5} N$ omits $\left\{F(a)<x<F\left(a_{1}\right): a_{0}<c_{*}<a_{1} \leq a_{*}\right.$ and $\left.\left\{a_{0}, a_{1}\right\} \subseteq M_{\mathbf{a}}\right\}$.

By renaming, without loss of generality the set of elements of $N$ is a countable ordinal, so we can finish the proof of the claim.

Discussion 3.9. 1) Note that a natural approach is to approximate the type of $c_{*}$ by formulas $\varphi(x)$ with parameters from $M$ such that $\varphi(x) \vdash$ " $x<a_{*}$ " and $M_{\mathbf{a}}$ "think" $\left|\varphi\left(M_{\mathbf{a}}\right)\right|$ is large enough than $b_{*}$. Then for $\sigma(x) \in \Sigma_{\mathbf{a}}$ (i.e. a term with parameters from $M$ ), which maps $\left\{c: c<_{M} a_{*}\right\}$ into $\left\{d: d<_{M} b_{*}\right\}$ we have to ensure $\sigma\left(c_{*}\right)$ will not realize the undesirable type, so it is natural to "shrink" $\varphi(x)$ to $\varphi^{\prime}(x)$ such that " $\left|\varphi^{\prime}(M)\right|$ is large enough then $|\varphi(M)| / b_{*}$ " in the sense of $M$ and $\sigma(-)$ is constant on $\varphi^{\prime}(M)$. This requires that also $|\varphi(M)| / b_{*}$ is large so a natural choice for " $\varphi(x)$ is large" means, e.g. $M \models "|\varphi(M)| \geq b_{*}^{n}$ " for every $n \in \mathbb{N}$. This is fine if $\neg\left(a_{*} E_{M}^{4} b_{*}\right)$, but not if we know $\neg\left(a_{*} E_{M}^{3} b_{*}\right)$ but possibly $a_{*} E_{M}^{4} b_{*}$. This motivates the main definition of $\mathbb{P}$ below.

2) We shall say that $\left(\varphi_{1}, \varphi_{2}\right) \in \mathbb{P}$ is a "poor man's substitute" to the original problem when:

(a) $\left[0, a_{*}\right)_{M_{\mathrm{a}}}$ is replaced by $\varphi_{1}(M)$

(b) $\left[0, b_{*}\right)_{M_{\mathrm{a}}}$ is replaced by $\varphi_{2}(M)$

(c) $F\left\lceil\left[0, a_{*}\right)_{M_{\mathbf{a}}}\right.$ is replaced by $F\left\lceil\varphi_{1}\left(M_{\mathbf{a}}\right)\right.$, really rounded to $\varphi_{2}(M)$

(d) $a_{*}>b_{*} \wedge \neg\left(a_{*} E_{M_{\mathrm{a}}}^{3}, b_{*}\right)$ is replaced by $\xi\left(\varphi_{1}\right)>\xi\left(\varphi_{2}\right)$, see $\boxplus_{1}(e)$ in the proof.

3) Why we demand condition $(\delta)$ of $\boxplus_{2}(a)$ in the proof of 3.7?

Assume $M_{\mathbf{a}} \prec M$ and $a \in M \backslash M_{\mathbf{a}}, a<_{M} a_{*}$ and $F^{+}$is an automorphism of $M \uparrow\{<\}$ extending $F$ then $\left\{f\left(a_{1}\right): a_{1}<_{M} a_{*}, a_{1}<_{M} a\right\},\left\{F\left(a_{2}\right): a_{2} \in M_{\mathbf{a}}, a_{*}<\right.$ $\left.\left.a_{2}<a_{*}\right\}\right)$ is a cut of $M_{\mathbf{a}}$, which $F^{+}(a)$ realizes in $M$. If $M$ "thinks" $\left|\varphi_{2}(M)\right|$ is $\ll$ $b_{*}, F$ may be one-to-one from $\varphi_{2}(M)$ onto some definable subset $\varphi_{2}^{\prime}(M) \subseteq\left[0, b_{*}\right)_{M}$. A reasonable suggestion is to demand $\left|\varphi_{2}(M)\right| \gg b_{*}$. Consider for transparency the case $M_{\mathbf{a}} \models$ " $a_{*}<b_{*} b_{*}$ ".

But then let $E$ be the definable convex equivalent relation on $\left[0, a_{*}\right)$ such that each equivalence class is of size $b_{* *}$, then the cut the new element realizes is really a cut of $\left[0, a_{*}\right) / E$. Now $F^{+}$maps every $E$-equivalence class to some $E^{\prime}$-equivalence class, $E^{\prime}$ a definable convex equivalence relation on $\left[0, b_{* *}\right)$ and $F$ as a map from $\left[0, a_{*}\right) / E$ into $\left[0, b_{*}\right) / E^{\prime}$ is defined, possible if $\left|\left[0, a_{*}\right) / E\right|=\left|\left[0, b_{*}\right) / E^{\prime}\right|$. 
The solution is via clause $(\delta)$, which tells us that in part (1),(2) of the discussion, $\xi\left(\varphi_{1}\right)>\xi\left(\varphi_{2}\right)$ is a real substitute, see clause (d) in part (2).

4) Why clause $(\gamma)$ in $\boxplus_{2}(a)$, defining $\mathbb{P}$ ? Otherwise $\varphi_{2}(-)$ may be irrelevant to the type we like to omit, so impossible.

5) By such approximations, i.e. member of $\mathbb{P}$,

(A) why can we arrive to a complete type?

Answer: As if we divide $\varphi_{1}$ to two sets at least one has the same $\xi(-)$ :

$(B)$ why can we continue to omit $p(x) \in \Gamma_{\mathbf{a}}$ ?

Answer: As if $\sigma(-)$ is a definable (in $M_{\mathbf{a}}$ ) function with domain $\varphi_{1}$ let $d_{*}$ be maximal such that $\left|\left\{a \in \varphi_{1}(M): \sigma(a)<d_{*}\right\}\right| \leq \frac{1}{2}\left|\varphi_{2}(M)\right|$, i.e. is in the middle in the right sense.

If $\sigma^{-1}\left\{d_{*}\right\}$ is large enough we easily finish; otherwise for some $n$ we have $d_{*} \notin$ $\left(a_{p, n}, b_{p, n}\right)$, so $\varphi_{1}(M) \wedge \sigma(x) \notin\left(a_{p, n}, b_{p, n}\right)$ has $\geq \frac{1}{2}\left(\varphi_{2}(M)\right)$ elements

$(C)$ why can guarantee that such $\sigma(x)$ does not realize the forbidden new type?

Answer: This is a major point. If $\xi\left(\varphi_{1}\right)>2 \xi\left(\varphi_{2}\right)$ this is easy (as in the case we use $\left.\neg a_{*} E_{M}^{4} b_{*}\right)$ and if for some $a_{1}<a_{2}$ we have $\xi\left(\varphi_{2}^{\prime}\right)>\xi\left(\varphi_{2}^{\prime}\right)$ we let

$$
\varphi_{2}^{\prime}(x)=\left(\varphi_{2}(x) \wedge a_{1} \leq x<a_{2} \wedge \sigma(x) \notin\left(F\left(a_{1}\right), F\left(a_{2}\right)\right)\right.
$$

and we let

$$
\varphi_{2}^{\prime \prime}(x):=\left(\varphi_{2} \wedge F\left(a_{1}\right) \leq x<F\left(a_{2}\right)\right)
$$

we are done, so assume there are no such $a_{1}, a_{2}$.

We consider two possible reasons for the "failure" of a suggested pair $\left(a_{1}, a_{2}\right)$. One reason is that maybe the length of the interval $\left[F\left(a_{1}\right), F\left(a_{2}\right)\right)$ of $\varphi_{2}\left(M_{1}\right)$ is too large. The second is that it is small enough but $\sigma(-)$ maps the large majority of $\varphi_{1}(M) \cap\left[a_{1}, a_{2}\right)$ into $\left[F\left(a_{1}\right), F\left(a_{2}\right)\right)$. In the second version we can define a version of it's property satisfied by $\left(a_{1}, a_{2}, F\left(a_{1}\right), F\left(a_{2}\right)\right)$. So we have enough intervals of pseudo second kind (pseudo means using the definable version of the property). So dividing $\varphi_{1}(M)$ to convex subsets of equal (suitable) size (essentially $a_{*}^{\xi\left(\varphi_{2}\right)}, \zeta \in \mathbb{R}_{>0}$ small enough) by $\left\langle a_{i}: i<i(*)\right\rangle$ we have: for some such interval $\left[a_{i}, a_{i+1}\right)$ there are $b_{1}, b_{2}$ as above. For those for which we cannot define $\left(F\left(a_{1}\right), F\left(a_{2}\right)\right)$ we can define it up to a good approximation. If there are enough, (this may include "pseudo cases" in respect to $F$ ) we can replace $\varphi_{1}(M)$ by $\varphi_{1}^{\prime}(M)=\left\{a_{i}: i<i(*)\right\}$ and $\varphi_{2}^{\prime}(-)$ defined by the function above.

So $\left|\varphi^{\prime}(M)\right|$ is significantly smaller than $\left|\varphi_{1}(M)\right|$, essentially $\xi\left(\varphi_{1}^{\prime}\right)=\xi\left(\varphi_{1}\right)-$ $\xi\left(a_{i+1}-a_{i}\right) \sim \xi\left(\varphi_{1}\right)-\xi\left(\varphi_{2}\right)+\zeta, \zeta$ quite small. But we are over-compensating so we decrease $\varphi_{2}(x)$ to $\varphi_{2}^{\prime}(x)$ which is quite closed to $\left\{F\left(a_{i}\right):\left[a_{i}, a_{i+1}\right)\right.$ is of the pseudo second kind $\}$ and $\xi\left(\varphi_{2}^{\prime}\right)$ is essentially $\xi\left(\varphi_{2}\right)-\xi\left(\varphi_{2}\right)+\zeta \sim \zeta$. So both lose similarly in the $\xi(-)$ measure but now, if we have arranged the numbers correctly $\xi\left(\varphi_{2}^{\prime}\right)>2 \xi\left(\varphi_{2}^{\prime}\right)$, a case we know to solve.

If there are not enough $i$ 's of the pseudo second kind, the function essentially inflates the image getting a finite cardinality arithmetic contradiction.

Theorem 3.10. Assume $\diamond_{\aleph_{1}}$. If $M$ is a countable model of PA, then $M$ has an elementary extension $N$ of cardinality $\aleph_{1}$ such that $E_{N}^{5}=E_{N}^{3}$, i.e. is 3-o.r. 
Proof. Without loss of generality $M$ has universe a countable ordinal. As we are assuming $\nabla_{\aleph_{1}}$, we choose $F_{\alpha}$ a partial function from $\alpha$ to $\alpha$ for $\alpha<\aleph_{1}$, i.e. $\bar{F}=\left\langle F_{\alpha}, \alpha<\aleph_{1}\right\rangle$ such that for every partial function $F: \aleph_{1} \rightarrow \aleph_{1}$, for stationarily many countable limit ordinals $\delta$ we have $F_{\delta}=F\lceil\delta$.

We now choose $\mathbf{a}_{\alpha} \in \mathrm{AP}_{\aleph_{0}}$ by induction on $\alpha<\aleph_{1}$ such that

(a) $\bullet \quad M_{\mathbf{a}_{0}}=M$

- $\Gamma_{\mathbf{a}_{0}}=\emptyset$

(b) $\left\langle\mathbf{a}_{\beta}: \beta \leq \alpha\right\rangle$ is $\leq_{\mathrm{AP}}$-increasing continuous

(c) if $\alpha=\delta+1, \delta$ is a countable limit ordinal, $M_{\mathbf{a}_{\delta}}$ has universe $\delta$ and for some $a_{\delta}, b_{\delta}$ the tuple $\left(\mathbf{a}_{\delta}, a_{\delta}, b_{\delta}, F_{\delta}\right)$ satisfies the assumptions of 3.7 on $\left(\mathbf{a}, a_{*}, b_{*}, F\right)$, they are necessarily unique (see $3.7(\mathrm{~A})(\mathrm{c})$ ), then $\mathbf{a}_{\delta+1}$ satisfies its conclusion (for some $c_{\delta}$ ).

Why can we carry the induction?

For $\alpha=0$ recall clause (a).

For $\alpha=1$, as $\Gamma_{\mathbf{a}_{0}}=\emptyset$ let $M_{\mathbf{a}_{1}}$ be a countable model such that $M=M_{\mathbf{a}_{0}} \prec$ $M_{\mathbf{a}_{1}}, M \neq M_{\mathbf{a}_{1}}$ and without loss of generality the universe of $M_{\mathbf{a}_{1}}$ is a countable ordinal.

Lastly, let $\Gamma_{\mathbf{a}_{1}}=\emptyset$.

For $\alpha$ a limit ordinal use 3.6 (2), i.e. choose the union, this is obvious.

For $\alpha=\beta+1$, if clause (c) apply use Claim 3.7 .

For $\alpha=\beta+1>1$ when clause (c) does not apply, this is easier than 3.7 (or choose $\left(a_{*}, h_{*}, F\right)$ such that $\left(\mathbf{a}_{\beta}, a_{*}, b_{*}, F\right)$ are as in the assumption 3.7 this is possible because $M_{\mathbf{a}_{\beta}}$ is non-standard, see the case $\alpha=1$, and note that $a, b \in$ $M_{\mathbf{a}_{\beta}} \backslash \mathbb{N} \Rightarrow a E_{M_{\mathbf{a}}}^{5} b$ because $M_{\mathbf{a}}$ is countable; so we can use 3.7).

Having carried the induction let $N=\cup\left\{M_{\mathbf{a}_{\alpha}}: \alpha<\aleph_{1}\right\}$.

Clearly $N$ is a model of $T$ of cardinality $\aleph_{1}$. We know that $E_{N}^{3} \subseteq E_{N}^{5}$ by 2.4. Toward contradiction assume $a_{*} E_{N}^{5} b_{*}$ but $\neg\left(a_{*} E_{N}^{3} b_{*}\right)$ where $a_{*}, b_{*} \in N \backslash M$. Without loss of generality $b_{*}<a_{*}$ and let $F$ be an order-isomorphism from $N_{<a_{*}}$ onto $N_{<b_{*}}$. So $S=\left\{\delta: F\left\lceil\delta=F_{\delta}\right\}\right.$ is stationary and $E=\left\{\delta: a_{*}, b_{*} \in M_{\mathbf{a}_{\delta}}, M_{\mathbf{a}_{\delta}}\right.$ has universe $\delta$ and $F$ maps $M_{<a_{*}}^{\mathbf{a}_{\delta}}$ onto $\left.M_{<b_{*}}^{\mathbf{a}_{\delta}}\right\}$ is a club of $\aleph_{1}$.

Choose $\delta \in S \cap E$ and use the choice of $\mathbf{a}_{\delta+1}$, i.e. clause (c) to get a contradiction.

Theorem 3.11. Assume $\lambda=\lambda^{<\lambda}$ and $\diamond_{S}$ where $S=S_{\lambda}^{\lambda^{+}}=\left\{\delta<\lambda^{+}: \operatorname{cf}(\delta)=\lambda\right\}$. For any model $M$ of $\mathrm{PA}$ there is a $\lambda$-saturated model $N$ of $\operatorname{Th}(M)$ of cardinality $\lambda^{+}$such that $E_{N}^{5} \subseteq E_{N}^{4}$.

Proof. Similar to 3.10 only the parallel of 3.7 is much easier.

Conjecture 3.12. 1) Assume $\lambda$ is strong limit singular of cofinality $\aleph_{0}$ and $\nabla_{S}$ where $S=S_{\aleph_{0}}^{\lambda^{+}}=\left\{\delta<\lambda^{+}: \operatorname{cf}(\delta)=\aleph_{0}\right\}$ and $\square_{\lambda}$. If $M$ is a model of PA, then $\operatorname{Th}(M)$ has a $\lambda$-universal model $N$ of cardinality $\lambda^{+}$which is 3 -o.r.

2) Any model $M$ of PA has a 3-o.r. elementary extension. 


\section{$\S$ 4. WEAKER VERSION OF PA}

We may wonder what is the weakest version of PA needed in the various results so below we define some variants and then remark when they suffice. But say when we add the function $2^{x}$, we prefer to add to the vocabulary a new function symbol and the relevant axioms (rather than an axiom stating that some definition of it has those properties). So we shall comment what version of PA is needed in the results of $\S 1, \S 2$.

Convention 4.1. A model is a model of $\mathrm{PA}_{-4}$ (see below) of vocabulary $\tau_{\mathrm{PA}}$ if not said otherwise.

Definition 4.2. We define the first order theories $\mathrm{PA}_{\ell}$ for $\ell \in\{-1, \ldots,-4\}$ and let $\mathrm{PA}_{\ell}^{\text {com }}$ be the set of completions of $\mathrm{PA}_{\ell}$.

Let $\mathrm{PA}_{\ell}$ consist of the following first order sentences in the vocabulary $\{0,1,<$ $,+, \times\}$ of $\mathbb{N}$ :

(a) for $\ell \leq 4$, the obvious axioms of addition and product and order, that is axioms describing the non-negative parts of discrete ordered rings,

(b) if $\ell \leq 3$ we also add division with remainder by any $n \in \mathbb{N}$,

(c) if $\ell \leq 2$ also add division with remainder,

(d) if $\ell \leq 1$, we add a unary function $F_{2}$ written $2^{x}$ with the obvious axioms for $x \mapsto 2^{x}$, including $(\forall x)(\exists y)\left(2^{y} \leq x<2^{y+1}\right)$.

Claim 4.3. For $M, N$ are models of $\mathrm{PA}_{-4}$; we still have 1.5(1),(1A),(1B),(3),(4) and 1.7 and 2.11(1),(2),(3),(5) and 1.12(1),(2),(3).

Claim 4.4. Claim 1.5(2) holds when $M$ is a model of $\mathrm{PA}_{-3}$.

Proof. The only difference is why can we choose $c_{1}, c_{2}$ there?

Now if we assume $M \models \mathrm{PA}$ this is obvious, but we are assuming $M \models \mathrm{PA}_{-3}$, still we can divide $b-a$ by $n-1$ and then get $c_{1}$ and $m<n-1$ such that $b-a=(n-1) \times c_{2}+m$. Let $c_{2}=a-c_{1}$ so $b=a+(n-1) \times c_{2}+m=$ $c_{1}+n \times c_{2}+m$. We still have to justify using $a-c_{2}$, i.e. showing $c_{2} \leq a$, but otherwise $b-a=(n-1) \times c_{2}+m \geq(n-1) \times a+m$, i.e. $b \geq n \times a+m$, contradiction.

Theorem 4.5. If $M_{1}, M_{2}$ are models of $\mathrm{PA}_{-1}$ then 1.8 holds, i.e. if $M_{2}$ is 2-orderrigid and $M_{1}, M_{2}$ are order-isomorphic then $M_{1}, M_{2}$ are almost $\{<,+\}$-isomorphic.

Proof. As in 1.8 with the following minor additions:

- in the proof of $(*)_{3}$ we use $M_{2} \models \mathrm{PA}_{-4}$,

- in the proof of $(*)_{5}$ we use $M_{\ell} \models \mathrm{PA}_{-2}$,

- in the proof of $(*)_{0}$ we use $M_{2} \models \mathrm{PA}_{-2}$,

- in the proof of $(*)_{7}$ we use $M_{1} \models \mathrm{PA}_{-4}$,

- in the proof of $(*)_{8}, \circledast_{2}$ we use $M_{\ell} \models \mathrm{PA}_{-1}$.

Claim 4.6. 1) If $M \models \mathrm{PA}_{-3}$ then 1.12(4) holds.

2) If $M \models \mathrm{PA}_{-1}$ then $1.12(5)$ holds.

Proof. Straightforward. 
We may wonder (see Definition 1.3(2)).

Question 4.7. Is there a 2-o.r. model of $\mathrm{PA}_{-1}$ ?

\section{REFERENCES}

[GT06] Rüdiger Göbel and Jan Trlifaj, Approximations and endomorphism algebras of modules, de Gruyter Expositions in Mathematics, vol. 41, Walter de Gruyter, Berlin, 2006.

[KS06] R. Kossak and J. Schmerl, The structure of models of Peano arithmetic, Oxford University Press, 2006.

[Sh:384] Saharon Shelah, Compact logics in ZFC : Complete embeddings of atomless Boolean rings, Non structure theory, Ch X.

[Sh:757] , Quite Complete Real Closed Fields, Israel Journal of Mathematics 142 (2004), 261-272, math.LO/0112212

Einstein Institute of Mathematics, Edmond J. Safra Campus, Givat Ram, The HeBrew University of Jerusalem, Jerusalem, 91904, Israel, and, Department of Mathematics, Hill Center - Busch Campus, Rutgers, The State University of New Jersey, 110 Frelinghuysen Road, Piscataway, NJ 08854-8019 USA

E-mail address: shelah@math.huji.ac.il

$U R L:$ http://shelah.logic.at 